\title{
Synthesis of Polycyclic Aromatics and Heteroaromatics via Electrophilic Cyclization
}

\author{
Tuanli Yao, Marino A. Campo and Richard C. Larock* \\ Department of Chemistry, Iowa State University, Ames, Iowa 50011
}

$\begin{array}{lc}\text { General } & \text { Page } \\ \text { Preparation of 2-(arylethynyl)biphenyls } & \text { S2-S2 } \\ \text { Electrophilic cyclization of 2-(arylethynyl)biphenyls by ICl } & \text { S2-S7 } \\ \text { Electrophilic cyclization of 2-(arylethynyl)biphenyls by } \mathrm{I}_{2} & \mathrm{~S} 7-\mathrm{S} 12 \\ \text { Electrophilic cyclization of 2-(arylethynyl)biphenyls by NBS } & \mathrm{S} 12-\mathrm{S} 12 \\ \begin{array}{l}\text { Electrophilic cyclization of 2-(arylethynyl)biphenyls by } \\ \text { PhSeCl }\end{array} & \mathrm{S} 12-\mathrm{S} 13 \\ \text { References } & \mathrm{S} 14-\mathrm{S} 15 \\ \text { Copies of }{ }^{1} \mathrm{H} \text { and }{ }^{13} \mathrm{C} \text { NMR spectra } & \mathrm{S} 16-\mathrm{S} 16 \\ & \mathrm{~S} 17-\mathrm{S} 60\end{array}$




\section{Supporting Information}

General. ${ }^{1} \mathrm{H}$ and ${ }^{13} \mathrm{C}$ NMR spectra were recorded at 300 and $75 \mathrm{MHz}$. Thin-layer chromatography was performed using commercially prepared 60-mesh silica gel plates (Whatman K6F), and visualization was effected with short wavelength UV light (254 nm) and a basic $\mathrm{KMnO} 4$ solution $\left[3 \mathrm{~g}\right.$ of $\mathrm{KMnO}_{4}+20 \mathrm{~g}$ of $\mathrm{K}_{2} \mathrm{CO}_{3}+5 \mathrm{~mL}$ of $\mathrm{NaOH}(5 \%)+300$ $\mathrm{mL}$ of $\mathrm{H}_{2} \mathrm{O}$ ]. All melting points are uncorrected. All reagents were used directly as commercially obtained unless otherwise noted. Compounds 1, 2, 6-17, 32-37, and 49-56 were reported in our earlier communication. ${ }^{1}$

General procedure for preparation of the 2-(arylethynyl)biphenyls. To a solution of the corresponding aryl iodide $(1.0 \mathrm{mmol})$ and the terminal alkyne $(1.2 \mathrm{mmol}, 1.2$ equiv) in $\mathrm{Et}_{3} \mathrm{~N}(4 \mathrm{~mL})$, were added $\mathrm{PdCl}_{2}\left(\mathrm{PPh}_{3}\right)_{2}(14 \mathrm{mg}, 2 \mathrm{~mol} \%)$ and $\mathrm{CuI}(2 \mathrm{mg}, 1 \mathrm{~mol}$ $\%)$. The resulting mixture was then heated under an $\mathrm{N}_{2}$ atmosphere at $55^{\circ} \mathrm{C}$ for $3 \mathrm{~h}$. The mixture was allowed to cool to room temperature, and the ammonium salt was removed by filtration. The solvent was removed under reduced pressure and the residue was purified by column chromatography on silica gel to afford the corresponding product.

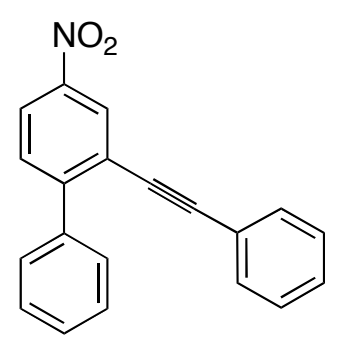

18

2-Phenylethynyl-4-nitrobiphenyl (18). 2-Iodo-4-nitrobiphenyl and phenylacetylene were employed. Purification by flash chromatography (10:1 hexane/EtOAc) afforded $254 \mathrm{mg}(85 \%)$ of the product as a yellow solid: $\mathrm{mp} 129-130{ }^{\circ} \mathrm{C} ;{ }^{1} \mathrm{H} \mathrm{NMR}\left(\mathrm{CDCl}_{3}\right) \delta 7.31$ - 
$7.38(\mathrm{~m}, 5 \mathrm{H}), 7.48-7.55(\mathrm{~m}, 3 \mathrm{H}), 7.58(\mathrm{~d}, J=8.7 \mathrm{~Hz}, 1 \mathrm{H}), 7.67-7.71(\mathrm{~m}, 2 \mathrm{H}), 8.21(\mathrm{dd}, J$

$=8.7,2.4 \mathrm{~Hz}, 1 \mathrm{H}), 8.50(\mathrm{~d}, J=2.4 \mathrm{~Hz}, 1 \mathrm{H}) ;{ }^{13} \mathrm{C} \mathrm{NMR}\left(\mathrm{CDCl}_{3}\right) \delta$ 87.4, 94.7, 122.6, 123.2, 123.5, 128.0, 128.5, 128.7, 129.1, 129.2, 129.4, 130.6, 131.8, 138.7, 147.0, 150.0; IR (neat, $\mathrm{cm}^{-1}$ ) 3630, 1514, 1343; HRMS Calcd for $\mathrm{C}_{20} \mathrm{H}_{13} \mathrm{NO}_{2}$ : 299.0946. Found: 299.0950.

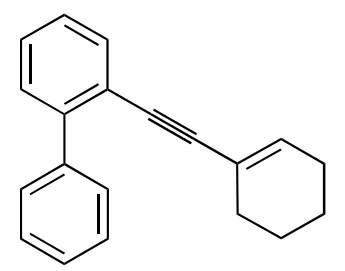

20

2-(Cyclohex-1-en-1ylethynyl)biphenyl (20). 2-Iodobiphenyl and 1-

ethynylcyclohexene were employed. Purification by flash chromatography (40:1 hexane/EtOAc) afforded $146 \mathrm{mg}(70 \%)$ of the product as a clear liquid: ${ }^{1} \mathrm{H}$ NMR $\left(\mathrm{CDCl}_{3}\right) \delta$ 1.53-1.65 (m, 4H), 2.07-2.13 (m, 4H), 6.03-6.06 (m, 1H), 7.26-7.47 (m, 6H), 7.53-7.57 (m, 1H), 7.62-7.66 (m, 2H); ${ }^{13} \mathrm{C}$ NMR $\left(\mathrm{CDCl}_{3}\right) \delta$ 21.8, 22.5, 26.0, 29.0, 86.9, $94.4,121.2,122.3,127.2,127.5,128.0,128.2,129.59,129.61,133.0,135.1,140.9$, 143.7; IR (neat, $\mathrm{cm}^{-1}$ ) 3059, 3023, 2931, 2199, 1475; HRMS Calcd for $\mathrm{C}_{20} \mathrm{H}_{18}: 258.1409$. Found: 258.1412 .

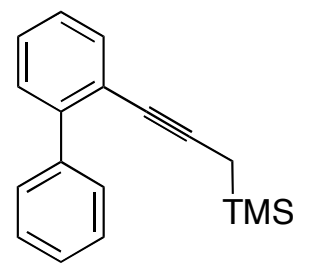

26

[3-(Biphenyl-2-yl)prop-2-ynyl](trimethyl)silane (26). 2-Iodobiphenyl and prop-2ynyl(trimethyl)silane were employed. Purification by flash chromatography (30:1 
hexane/EtOAc) afforded $128 \mathrm{mg}(49 \%)$ of the product as a clear liquid: ${ }^{1} \mathrm{H}$ NMR $\left(\mathrm{CDCl}_{3}\right) \delta 0.00(\mathrm{~s}, 9 \mathrm{H}), 1.59(\mathrm{~s}, 2 \mathrm{H})$, 7.24-7.34 (m, 4H), 7.37-7.41 (m, 2H), 7.48-7.51 (m, $1 \mathrm{H})$, 7.54-7.58 (m, 2H); ${ }^{13} \mathrm{C} \mathrm{NMR}\left(\mathrm{CDCl}_{3}\right) \delta$-1.9, 8.3, 78.9, 91.5, 123.2, 126.9, 127.1, 127.2, 127.9, 129.3, 129.5, 133.2, 141.2, 143.4; IR (neat, $\mathrm{cm}^{-1}$ ) 3060, 2955, 2205, 1476, 1249; HRMS Calcd for $\mathrm{C}_{18} \mathrm{H}_{20} \mathrm{Si}$ : 264.1334. Found: 264.1339 .

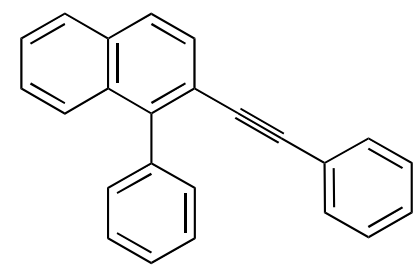

28

1-Phenyl-2-(phenylethynyl)naphthalene (28). 2-Iodo-1-phenylnaphthalene and phenylacetylene were employed. Purification by flash chromatography (40:1 hexane/EtOAc) afforded $301 \mathrm{mg}(99 \%)$ of the product as a yellow oil: ${ }^{1} \mathrm{H} \mathrm{NMR}\left(\mathrm{CDCl}_{3}\right)$ ठ 7.16-7.21 (m, 2H), 7.23-7.27 (m, 3H), 7.38-7.44 (m, 1H), 7.47-7.55 (m, 5H), 7.65-7.70 (m, 2H), 7.82-7.89 (m, 2H); ${ }^{13} \mathrm{C} \mathrm{NMR}\left(\mathrm{CDCl}_{3}\right) \delta 90.2,93.4,120.4,123.7,126.6,126.7$, $126.9,127.68,127.7,128.18,128.22,128.23,128.4,128.6,130.9,131.6,132.4,133.4$, 139.2, 143.3; IR (neat, $\mathrm{cm}^{-1}$ ) 3056, 1950, 1598, 1505, 1490; HRMS Calcd for $\mathrm{C}_{24} \mathrm{H}_{16}$ : 304.1252. Found: 304.1257.

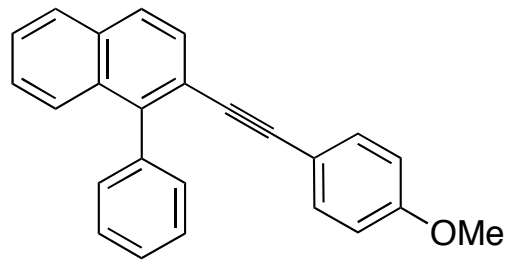

30

2-[(4-Methoxyphenyl)ethynyl]-1-phenylnaphthalene (30). 2-Iodo-1-

phenylnaphthalene and $p$-methoxyphenyl acetylene were employed. Purification by flash 
chromatography (20:1 hexane/EtOAc) afforded $276 \mathrm{mg}(82 \%)$ of the product as a white solid: mp 109-111 ${ }^{\circ} \mathrm{C} ;{ }^{1} \mathrm{H}$ NMR $\left(\mathrm{CDCl}_{3}\right) \delta 3.81(\mathrm{~s}, 3 \mathrm{H}), 6.82(\mathrm{dd}, J=2.1,6.9 \mathrm{~Hz}, 2 \mathrm{H})$, $7.17(\mathrm{dd}, J=2.1,6.9 \mathrm{~Hz}, 2 \mathrm{H}), 7.34-7.57(\mathrm{~m}, 7 \mathrm{H}), 7.68-7.74(\mathrm{~m}, 2 \mathrm{H}), 7.84-7.92(\mathrm{~m}, 2 \mathrm{H})$; ${ }^{13} \mathrm{C}$ NMR $\left(\mathrm{CDCl}_{3}\right) \delta 55.4,88.9,93.5,114.1,115.7,120.7,126.4,126.6,126.8,127.60$, 127.62, 128.1, 128.2, 128.4, 130.9, 132.4, 133.0, 133.1, 139.3, 142.8, 159.7; IR (neat, $\mathrm{cm}^{-}$ 1) $3055,2956,2836,2207,1605,1511$; HRMS Calcd for $\mathrm{C}_{25} \mathrm{H}_{18} \mathrm{O}: 334.1358$. Found: 334.1365 .

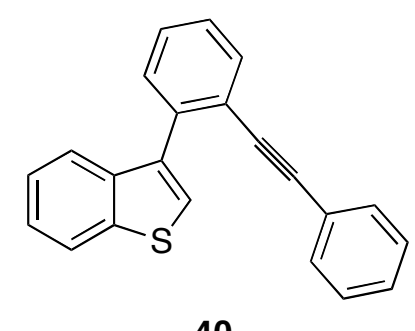

40

3-[2-(Phenylethynyl)phenyl]-benzothiophene (40). To a solution of 2-bromophenyl phenyl acetylene (1.5 mmol, $386 \mathrm{mg}$ ) and 1-benzothien-3-ylboronic acid (320 mg, 1.2 equiv) in $7.5 \mathrm{~mL}$ of DME were added $\mathrm{Pd}(\mathrm{dba})_{2}(43.2 \mathrm{mg}, 5 \mathrm{~mol} \%), \mathrm{PPh}_{3}(39 \mathrm{mg}, 10 \mathrm{~mol}$ $\%$ ) and $\mathrm{CsF}$ (456 mg, 2.0 equiv). The resulting mixture was heated under an $\mathrm{N}_{2}$ atmosphere at $100{ }^{\circ} \mathrm{C}$ for $24 \mathrm{~h}$. The mixture was cooled to room temperature and diluted with $70 \mathrm{~mL}$ of ether, washed with $25 \mathrm{~mL}$ of satd $\mathrm{NaCl}$, dried $\left(\mathrm{MgSO}_{4}\right)$ and filtered. The solvent was evaporated under reduced pressure and the residue was chromatographed using 50:1 hexane/EtOAc to afford $145 \mathrm{mg}(31 \%)$ of the product as a yellow oil: ${ }^{1} \mathrm{H}$

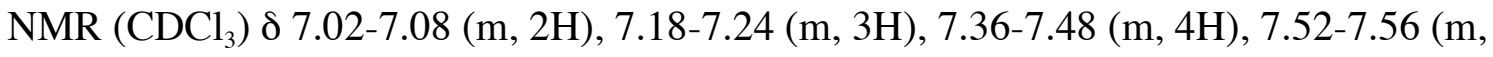
1H), 7.63 (s, 1H), 7.70-7.74 (m, 1H), 7.76-7.80 (m, 1H), 7.94-7.98 (m, 1H); ${ }^{13} \mathrm{C}$ NMR $\left(\mathrm{CDCl}_{3}\right) \delta 89.3,93.3,122.9,123.3,123.4,123.8,124.3,124.5,125.6,127.8,128.3$ 
128.4, 128.5, 130.3, 131.5, 132.9, 136.5, 138.3, 138.7, 140.2; IR (neat, $\mathrm{cm}^{-1}$ ) 3057, 1597, 1492, 1441; HRMS Calcd for $\mathrm{C}_{22} \mathrm{H}_{14} \mathrm{~S}: 310.0816$. Found: 310.0821.

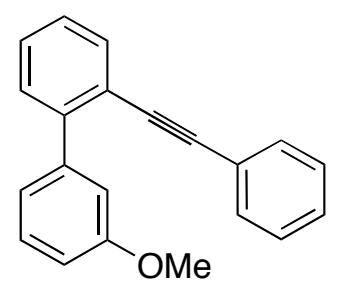

43

3'-Methoxy-2-(phenylethynyl)biphenyl (43). This alkyne was prepared from 2(phenylethynyl)phenylboronic acid and 3-iodoanisole by following the same procedure as compound 40 at $80{ }^{\circ} \mathrm{C}$. 2-(Phenylethynyl)phenylboronic acid (133 mg, $\left.0.6 \mathrm{mmol}\right), 1$ methoxy-3-iodobenzene (126 mg, 0.9 equiv), $\mathrm{Pd}(\mathrm{dba})_{2}\left(14.4 \mathrm{mg}, 0.05\right.$ equiv), $\mathrm{PPh}_{3}(13$ $\mathrm{mg}, 0.1$ equiv), $\mathrm{CsF}$ (182 mg, 2.0 equiv) and $\mathrm{DME}(2.5 \mathrm{~mL})$ afforded, after purification by flash column chromatography (silica gel, 20:1 hexane/EtOAc), $76 \mathrm{mg}(50 \%)$ of the indicated compound 43 as a light yellow oil: ${ }^{1} \mathrm{H}$ NMR $\left(\mathrm{CDCl}_{3}\right) \delta 3.86(\mathrm{~s}, 3 \mathrm{H}), 6.97-7.00$ (m, 1H), 7.26-7.33 (m, 5H), 7.36-7.49 (m, 6H), 7.67-7.71 (m, 1H); ${ }^{13} \mathrm{C} \mathrm{NMR}\left(\mathrm{CDCl}_{3}\right) \delta$ $55.5,89.6,92.7,113.7,115.0,121.8,122.2,123.7,127.4,128.4,128.5,128.8,129.2$, 129.7, 131.7, 133.2, 142.2, 144.0, 159.4; IR $\left(\right.$ neat, $\left.\mathrm{cm}^{-1}\right)$ 3058, 3023, 2955, 2936, 2833, 1599, 1581, 1490; HRMS Calcd for $\mathrm{C}_{21} \mathrm{H}_{16} \mathrm{O}: 284.1201$. Found: 284.1206.

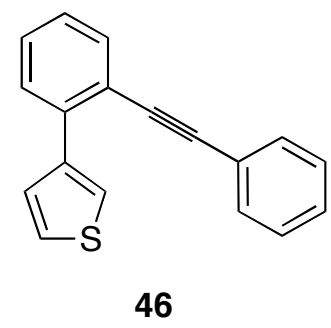

3-[2-(Phenylethynyl)phenyl]thiophene (46). This alkyne was prepared from 2bromophenyl phenyl acetylene and 3-thiopheneboronic acid by following the same 
procedure as compound 40 at $90{ }^{\circ} \mathrm{C}$. 1-Bromo-2-(phenylethynyl)benzene $(257 \mathrm{mg}, 1$ mmol), 3-thiopheneboronic acid (154 mg, 1.2 equiv), $\mathrm{Pd}(\mathrm{dba})_{2}$ (24 mg, 0.05 equiv), $\mathrm{PPh}_{3}$ (22 mg, 0.1 equiv), CsF (304 mg, 2.0 equiv) and DME (4 mL) afforded, after purification by flash column chromatography (silica gel, 20:1 hexane/EtOAc), $235 \mathrm{mg}(90 \%)$ of the indicated compound 46 as a light yellow oil: ${ }^{1} \mathrm{H}$ NMR $\left(\mathrm{CDCl}_{3}\right) \delta$ 7.26-7.39 $(\mathrm{m}, 6 \mathrm{H})$, 7.42-7.44 (m, 2H), 7.48-7.50 (m, 1H), 7.53 (dd, $J=0.9,3.9 \mathrm{~Hz}, 1 \mathrm{H}), 7.61-7.64(\mathrm{~m}, 1 \mathrm{H})$, $7.70(\mathrm{dd}, J=0.9,2.1 \mathrm{~Hz}, 1 \mathrm{H}) ;{ }^{13} \mathrm{C} \mathrm{NMR}\left(\mathrm{CDCl}_{3}\right) \delta 89.7,92.7,121.2,123.5,123.7,124.8$, 127.0, 128.3, 128.4, 128.6, 128.7, 129.1, 131.5, 133.3, 138.2, 141.0; IR (neat, $\left.\mathrm{cm}^{-1}\right) 3103$, 3058, 3028, 1597, 1492, 1442; HRMS Calcd for $\mathrm{C}_{18} \mathrm{H}_{12} \mathrm{~S}: 260.0660$. Found: 260.0663 .

\section{General procedure for the electrophilic cyclization of 2-(arylethynyl)biphenyls by}

ICl. To a solution of 2-(arylethynyl)biphenyl $(0.30 \mathrm{mmol})$ in $\mathrm{CH}_{2} \mathrm{Cl}_{2}(3 \mathrm{~mL})$ under $\mathrm{N}_{2}$ was added $\mathrm{ICl}$ (1.2 equiv) in $\mathrm{CH}_{2} \mathrm{Cl}_{2}(0.5 \mathrm{~mL})$ at $-78{ }^{\circ} \mathrm{C}$. The reaction mixture was stirred at $-78{ }^{\circ} \mathrm{C}$ for $1 \mathrm{~h}$ unless otherwise indicated. The reaction mixture was then diluted with diethyl ether $(50 \mathrm{~mL})$, washed with $25 \mathrm{~mL}$ of satd aq $\mathrm{Na}_{2} \mathrm{~S}_{2} \mathrm{O}_{3}$, dried $\left(\mathrm{MgSO}_{4}\right)$, and filtered. The solvent was evaporated under reduced pressure and the product was purified by chromatography on a silica gel column.

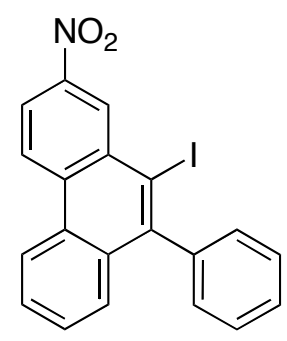

19

10-Iodo-2-nitro-9-phenylphenanthrene (19). Purification by flash chromatography (7:1 hexane/EtOAc) afforded $112 \mathrm{mg}(88 \%)$ of the product as a yellow solid: $\mathrm{mp} 182-$ $183{ }^{\circ} \mathrm{C} ;{ }^{1} \mathrm{H}$ NMR $\left(\mathrm{CDCl}_{3}\right) \delta$ 7.25-7.30 (m, 2H), 7.44-7.62 (m, 5H), $7.75(\mathrm{dt}, J=1.2,7.8$ 
$\mathrm{Hz}, 1 \mathrm{H}), 8.45(\mathrm{td}, J=2.7,9.0 \mathrm{~Hz}, 1 \mathrm{H}), 8.73(\mathrm{~d}, J=8.4 \mathrm{~Hz}, 1 \mathrm{H}), 8.81(\mathrm{dd}, J=3.3,9.3 \mathrm{~Hz}$, 1H), $9.42(\mathrm{t}, J=2.7 \mathrm{~Hz}, 1 \mathrm{H}) ;{ }^{13} \mathrm{C} \mathrm{NMR}\left(\mathrm{CDCl}_{3}\right) \delta 105.5,121.3,123.8,124.7,128.3$, $128.5,128.9,129.28,129.33,129.4,129.8,131.1,132.9,133.8,134.8,144.8,147.2$, 148.0; IR (neat, $\mathrm{cm}^{-1}$ ) 3080, 3059, 3025, 1577, 1515, 1345; HRMS Calcd for $\mathrm{C}_{20} \mathrm{H}_{12} \mathrm{INO}_{2}$ : 424.9913. Found: 424.9921.

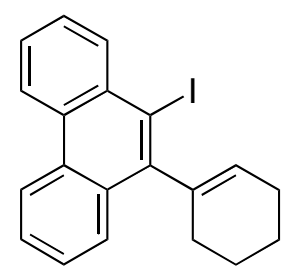

21

9-(Cyclohex-1-en-1-yl)-10-iodophenanthrene (21). Purification by flash chromatography (50:1 hexane/EtOAc) afforded $79 \mathrm{mg}(70 \%)$ of the product as a colorless oil: ${ }^{1} \mathrm{H}$ NMR $\left(\mathrm{CDCl}_{3}\right) \delta$ 1.85-2.01 (m, 4H), 2.19-2.26 (m, 1H), 3.35-2.48 (m, 3H), 5.71$5.74(\mathrm{~m}, 1 \mathrm{H}), 7.54-7.60(\mathrm{~m}, 1 \mathrm{H}), 7.62-7.70(\mathrm{~m}, 3 \mathrm{H}), 8.06(\mathrm{dd}, J=0.9,8.1 \mathrm{~Hz}, 1 \mathrm{H}), 8.41-$ $8.46(\mathrm{~m}, 1 \mathrm{H}), 8.61-8.64(\mathrm{~m}, 1 \mathrm{H}), 8.69(\mathrm{~d}, J=8.7 \mathrm{~Hz}, 1 \mathrm{H}) ;{ }^{13} \mathrm{C} \mathrm{NMR}\left(\mathrm{CDCl}_{3}\right) \delta 22.3$, $23.2,25.7,29.5,105.4,122.8,123.0,127.2,127.28,127.33,128.0,128.2,129.2,130.5$, 130.7, 131.4, 132.8, 134.5, 142.4, 147.2; IR $\left(\right.$ neat, $\left.\mathrm{cm}^{-1}\right)$ 3067, 3025, 2926, 1562, 1482, 1445; HRMS Calcd for $\mathrm{C}_{20} \mathrm{H}_{17} \mathrm{I}$ : 384.0375 . Found: 384.0380 .

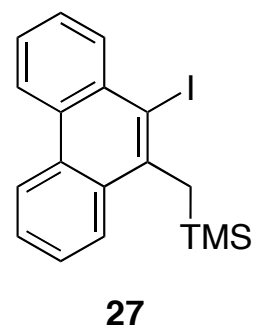

[(10-Iodo-9-phenanthryl)methyl](trimethyl)silane (27). Purification by flash chromatography (50:1 hexane/EtOAc) afforded $54 \mathrm{mg}(50 \%)$ of the product as a white 
solid: $\mathrm{mp} 70-72{ }^{\circ} \mathrm{C} ;{ }^{1} \mathrm{H}$ NMR $\left(\mathrm{CDCl}_{3}\right) \delta 0.11(\mathrm{~s}, 9 \mathrm{H}), 3.24(\mathrm{~s}, 2 \mathrm{H}), 7.56-7.71(\mathrm{~m}, 4 \mathrm{H})$, $8.09(\mathrm{dd}, J=0.8,8.1 \mathrm{~Hz}, 1 \mathrm{H}), 8.39-8.42(\mathrm{~m}, 1 \mathrm{H}), 8.58-8.62(\mathrm{~m}, 1 \mathrm{H}), 8.70-8.73(\mathrm{~m}, 1 \mathrm{H})$; ${ }^{13} \mathrm{C} \mathrm{NMR}\left(\mathrm{CDCl}_{3}\right) \delta 0.00,31.1,106.0,122.2,122.9,125.9,126.54,126.57,126.64$, 127.5, 129.2, 130.1, 130.7, 133.0, 134.2, 141.8; IR (neat, $\mathrm{cm}^{-1}$ ) 3068, 2951, 1562, 1485, 1445; HRMS Calcd for $\mathrm{C}_{18} \mathrm{H}_{19}$ ISi: 390.0301 . Found: 390.0310.

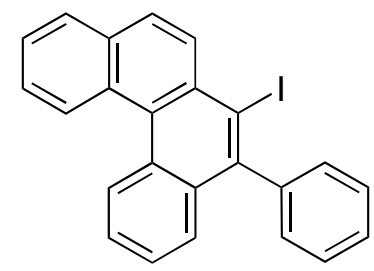

29

6-Iodo-5-phenylbenzo $[c]$ phenanthrene (29). Purification by flash chromatography (3:1 hexane $/ \mathrm{CH}_{2} \mathrm{Cl}_{2}$ ) afforded $61 \mathrm{mg}(48 \%)$ of the product as a white solid: $\mathrm{mp} 159-160$ ${ }^{\circ} \mathrm{C} ;{ }^{1} \mathrm{H}$ NMR $\left(\mathrm{CDCl}_{3}\right) \delta$ 7.32-7.36 (m, 2H), 7.44-7.47 (m, 1H), 7.52-7.60 (m, 4H), 7.62$7.70(\mathrm{~m}, 3 \mathrm{H}), 7.95(\mathrm{~d}, J=9.0 \mathrm{~Hz}, 1 \mathrm{H}), 8.03-8.07(\mathrm{~m}, 1 \mathrm{H}), 8.43(\mathrm{~m}, J=9.0 \mathrm{~Hz}, 1 \mathrm{H}), 9.04$ $(\mathrm{d}, J=8.1 \mathrm{~Hz}, 2 \mathrm{H}) ;{ }^{13} \mathrm{C} \mathrm{NMR}\left(\mathrm{CDCl}_{3}\right) \delta 106.4,126.4,126.6,126.7,126.8,128.1,128.3$, 128.4, 128.6, 128.67, 128.71, 128.8, 129.0, 129.6, 130.1, 130.3, 131.2, 132.2, 133.4, 133.8, 145.2, 145.4; IR (neat, $\mathrm{cm}^{-1}$ ) 3057, 1599, 1503, 1488; HRMS Calcd for $\mathrm{C}_{24} \mathrm{H}_{15} \mathrm{I}$ : 430.0219. Found: 430.0228

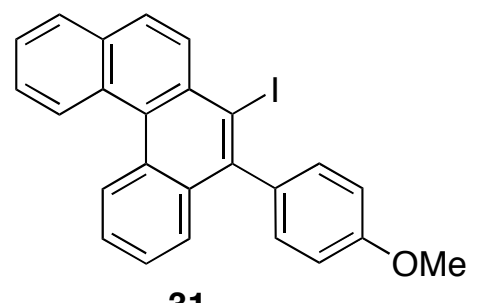

31

5-(4-Methoxyphenyl)-6-iodobenzo[c]phenanthrene (31). Purification by flash chromatography (20:1 hexane/ EtOAc) afforded $134 \mathrm{mg}$ (97\%) of the product as a green solid: $\mathrm{mp} 186-187^{\circ} \mathrm{C} ;{ }^{1} \mathrm{H}$ NMR $\left(\mathrm{CDCl}_{3}\right) \delta 3.94(\mathrm{~s}, 3 \mathrm{H}), 7.10(\mathrm{~d}, J=4.5 \mathrm{~Hz}, 2 \mathrm{H}), 7.23-$ 
$7.26(\mathrm{~m}, 2 \mathrm{H}), 7.42-7.47(\mathrm{~m}, 1 \mathrm{H}), 7.57-7.69(\mathrm{~m}, 4 \mathrm{H}), 7.94(\mathrm{~d}, J=9.0 \mathrm{~Hz}, 1 \mathrm{H}), 8.02-8.06$ (m, 1H), $8.42(\mathrm{~d}, J=9.0 \mathrm{~Hz}, 1 \mathrm{H}), 9.01-9.05(\mathrm{~m}, 2 \mathrm{H}) ;{ }^{13} \mathrm{C} \mathrm{NMR}\left(\mathrm{CDCl}_{3}\right) \delta 55.6,107.3$, 114.1, 126.4, 126.6, 126.7, 126.8, 128.41, 128.44, 128.61, 128.64, 128.8, 129.0, 129.7, 130.2, 131.3, 131.5, 132.4, 133.75, 133.80, 138.0, 145.0, 159.4; IR (neat, $\left.\mathrm{cm}^{-1}\right) 3065$, 2961, 2838, 1607, 1510, 1247; HRMS Calcd for $\mathrm{C}_{25} \mathrm{H}_{17} \mathrm{IO}: 460.0324$. Found: 460.0334 .

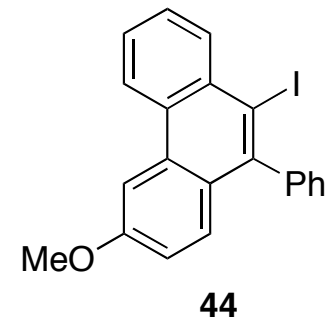

9-Iodo-3-methoxy-10-phenylphenanthrene (44). Purification by flash chromatography (30:1 hexane/ EtOAc) afforded $83 \mathrm{mg}(66 \%)$ of the product as a white solid: $\mathrm{mp} 136-138^{\circ} \mathrm{C} ;{ }^{1} \mathrm{H}$ NMR $\left(\mathrm{CDCl}_{3}\right) \delta 4.02(\mathrm{~s}, 3 \mathrm{H}), 7.05(\mathrm{dd}, J=2.5,9.3 \mathrm{~Hz}, 1 \mathrm{H})$, 7.26-7.35 (m, 3H), 7.51-7.58 (m, 3H), 7.68-7.71 (m, 2H), 8.09 (d, $J=2.4 \mathrm{~Hz}, 1 \mathrm{H}), 8.43-$ $8.47(\mathrm{~m}, 1 \mathrm{H}), 8.59-8.62(\mathrm{~m}, 1 \mathrm{H}) ;{ }^{13} \mathrm{C} \mathrm{NMR}\left(\mathrm{CDCl}_{3}\right) \delta$ 55.7, 103.3, 104.3, 117.0, 122.9, $127.3,127.5,128.0,128.4,128.7,130.2,130.4,130.5,132.0,133.0,134.9,145.3,145.7$, 158.9; IR (neat, $\mathrm{cm}^{-1}$ ) 3056, 3025, 2957, 2933, 2834, 1613, 1576, 1519; HRMS Calcd for $\mathrm{C}_{21} \mathrm{H}_{15} \mathrm{IO}: 410.0168$. Found: 410.0175 .<smiles>COc1cccc2c1c(-c1ccccc1)c(I)c1ccccc12</smiles>

45

9-Iodo-1-methoxy-10-phenylphenanthrene (45). Purification by flash chromatography (30:1 hexane/ EtOAc) afforded $26 \mathrm{mg}$ (20\%) of the product as a light 
yellow oil: ${ }^{1} \mathrm{H}$ NMR $\left(\mathrm{CDCl}_{3}\right) \delta 3.34(\mathrm{~s}, 3 \mathrm{H}), 6.94(\mathrm{~d}, J=7.8 \mathrm{~Hz}, 1 \mathrm{H}), 7.18-7.21(\mathrm{~m}, 2 \mathrm{H})$, 7.37-7.46 (m, 3H), 7.58-7.69 (m, 3H), $8.38(\mathrm{~d}, J=8.4 \mathrm{~Hz}, 1 \mathrm{H}), 8.48-8.52(\mathrm{~m}, 1 \mathrm{H}), 8.64-$ $8.67(\mathrm{~m}, 1 \mathrm{H}) ;{ }^{13} \mathrm{C} \mathrm{NMR}\left(\mathrm{CDCl}_{3}\right) \delta$ 56.2, 109.5, 110.0, 115.8, 123.4, 123.5, 126.5, 127.4, 127.6, 128.0, 128.5, 129.0, 130.4, 132.7, 133.0, 135.3, 142.9, 151.1, 156.6; IR (neat, $\mathrm{cm}^{-}$ 1) $3056,3021,2929,1601,1575,1455$; HRMS Calcd for $\mathrm{C}_{21} \mathrm{H}_{15} \mathrm{IO}: 410.0168$. Found: 410.0172 .

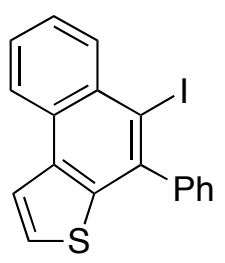

47

5-Iodo-4-phenylnaphtho[2,1-b]thiophene (47). Purification by flash chromatography (20:1 hexane/ EtOAc) afforded $58 \mathrm{mg}(50 \%)$ of the product as a light yellow solid: $\mathrm{mp}$ 98-99 ${ }^{\circ} \mathrm{C} ;{ }^{1} \mathrm{H}$ NMR $\left(\mathrm{CDCl}_{3}\right)$ d 7.41-7.44 (m, 2H), 7.54-7.58 (m, 4H), 7.63-7.68 (m, 2H), $8.01(\mathrm{~d}, J=5.4 \mathrm{~Hz}, 1 \mathrm{H}), 8.30-8.35(\mathrm{~m}, 1 \mathrm{H}), 8.43-8.49(\mathrm{~m}, 1 \mathrm{H}) ;{ }^{13} \mathrm{C} \mathrm{NMR}\left(\mathrm{CDCl}_{3}\right) \delta$ $101.18,122.2,124.2,127.3,127.4,127.9,128.8,128.9,129.0,129.6,132.8,134.4,136.3$, 139.7, 141.2, 144.8; IR (neat, $\mathrm{cm}^{-1}$ ) 3102, 3059, 3025, 1551, 1492, 1442; HRMS Calcd for $\mathrm{C}_{18} \mathrm{H}_{11} \mathrm{IS}: 385.9626$. Found: 385.9633 .

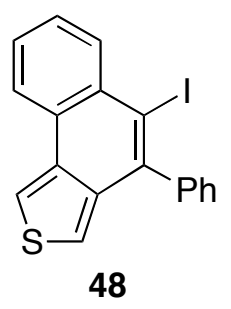

5-Iodo-4-phenylnaphtho[1,2-c] thiophene (48). Purification by flash chromatography (20:1 hexane/ EtOAc) afforded $43 \mathrm{mg}$ (37\%) of the product as a white solid: $\mathrm{mp}$ 149-150 ${ }^{\circ} \mathrm{C} ;{ }^{1} \mathrm{H}$ NMR $\left(\mathrm{CDCl}_{3}\right) \delta$ 7.36-7.40 (m, 2H), 7.52-7.67 (m, 6H), 7.81 (s, 1H), 8.12-8.17 (m, 
$1 \mathrm{H}), 8.40-8.44(\mathrm{~m}, 1 \mathrm{H}) ;{ }^{13} \mathrm{C} \mathrm{NMR}\left(\mathrm{CDCl}_{3}\right) \delta 101.1,121.4,124.0,127.6,127.7,128.1$, $129.0,129.4,132.9,133.8,134.5,135.2,138.2,140.4,144.1$ (one $\mathrm{sp}^{2}$ carbon missing due to overlap); IR (neat, $\mathrm{cm}^{-1}$ ) 3057, 3022, 1554, 1496; HRMS Calcd for $\mathrm{C}_{18} \mathrm{H}_{11}$ IS:

385.9626. Found: 385.9632 .

General procedure for the electrophilic cyclization of 2-(1-alkynyl)biphenyls by $\mathbf{I}_{2}$. To a solution of 2-(1-alkynyl)biphenyl $(0.30 \mathrm{mmol})$ in $\mathrm{CH}_{2} \mathrm{Cl}_{2}(3 \mathrm{~mL})$ was added $\mathrm{I}_{2}(3.0$ equiv) and $\mathrm{NaHCO}_{3}$ (3.0 equiv) at room temperature. The reaction mixture was stirred at room temperature for $24 \mathrm{~h}$ unless otherwise indicated. The reaction mixture was then diluted with diethyl ether $(50 \mathrm{~mL})$, washed with satd aq $\mathrm{Na}_{2} \mathrm{~S}_{2} \mathrm{O}_{3}(25 \mathrm{~mL})$, dried $\left(\mathrm{MgSO}_{4}\right)$, and filtered. The solvent was evaporated under reduced pressure and the product was purified by chromatography on a silica gel column.

9-Iodo-10-phenylphenanthrene (2). Purification by flash chromatography (50:1 hexane/EtOAc) afforded $92 \mathrm{mg}(80 \%)$ of the product as a white solid with a melting point and spectral properties identical to those previously reported. ${ }^{2}$

\section{General procedure for the electrophilic cyclization of 2-(1-alkynyl)biphenyls by}

NBS. To a solution of 2-(1-alkynyl)biphenyl $(0.30 \mathrm{mmol})$ in $\mathrm{CH}_{2} \mathrm{Cl}_{2}(3 \mathrm{~mL})$ was added NBS (1.2 equiv) and silica gel $(50 \mathrm{mg})$ at room temperature. The reaction mixture was stirred at room tempature for $144 \mathrm{~h}$ unless otherwise indicated. The reaction mixture was then diluted with diethyl ether $(50 \mathrm{~mL})$, washed with satd aq $\mathrm{Na}_{2} \mathrm{~S}_{2} \mathrm{O}_{3}(25 \mathrm{~mL})$, dried $\left(\mathrm{MgSO}_{4}\right)$, and filtered. The solvent was evaporated under reduced pressure and the product was purified by chromatography on a silica gel column. 


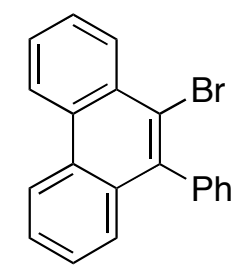

3

9-Bromo-10-phenylphenanthrene (3). Purification by flash chromatography (40:1 hexane/EtOAc) afforded $86 \mathrm{mg}(86 \%)$ of the product as a white solid: $\mathrm{mp} 108-109^{\circ} \mathrm{C}$; ${ }^{1} \mathrm{H}$ NMR $\left(\mathrm{CDCl}_{3}\right) \delta$ 7.34-7.38 (m, 2H), 7.41-7.47 (m, 2H), 7.50-7.60 (m, 3H), 7.64-7.77 $(\mathrm{m}, 3 \mathrm{H}), 8.53-8.57(\mathrm{~m}, 1 \mathrm{H}), 8.72-8.77(\mathrm{~m}, 2 \mathrm{H}) ;{ }^{13} \mathrm{C} \mathrm{NMR}\left(\mathrm{CDCl}_{3}\right) \delta 122.9,123.8,127.1$, $127.3,127.7,127.9,128.0,128.2,128.7,129.2,129.3,130.2,130.7,131.2,132.9,139.9$, 141.3; IR (neat, $\mathrm{cm}^{-1}$ ) 3070, 3058, 3027, 1583, 1567, 1484; HRMS Calcd for $\mathrm{C}_{20} \mathrm{H}_{15} \mathrm{Br}$ : 332.0201. Found: 332.0209.

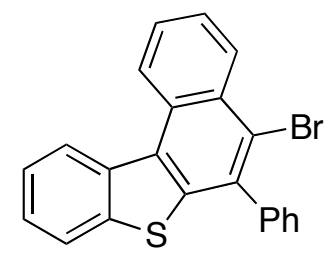

41

5-Bromo-6-phenylbenzo[b]naphtha[1,2- $d]$ thiophene (41). Purification by flash chromatography (40:1 hexane/EtOAc) afforded $102 \mathrm{mg}(88 \%)$ of the product as a yellow oil: ${ }^{1} \mathrm{H}$ NMR $\left(\mathrm{CDCl}_{3}\right) \delta$ 7.46-7.53 (m, 3H), 7.54-7.63 (m, 4H), 7.68-7.74 (m, 1H), 7.78$7.83(\mathrm{~m}, 1 \mathrm{H}), 7.88(\mathrm{dd}, J=7.8,0.6 \mathrm{~Hz}, 1 \mathrm{H}), 8.64(\mathrm{dd}, J=8.4,1.2 \mathrm{~Hz}, 1 \mathrm{H}), 8.86(\mathrm{~d}, J=$ 8.1 Hz, 1H), $9.07(\mathrm{~d}, J=7.8 \mathrm{~Hz}, 1 \mathrm{H}) ;{ }^{13} \mathrm{C} \mathrm{NMR}\left(\mathrm{CDCl}_{3}\right) \delta 122.7,123.3,123.6,125.1$, $125.3,125.8,126.5,127.9,128.9,129.0,129.70,129.74,130.9,131.0,136.4,136.6$, 140.6, 141.0, 141.4; IR (neat, $\mathrm{cm}^{-1}$ ) 3059, 2921, 1558, 1494, 1442; HRMS Calcd for $\mathrm{C}_{22} \mathrm{H}_{13} \mathrm{BrS}$ : 387.9921. Found: 387.9930. 
General procedure for the electrophilic cyclization of 2-(1-alkynyl)biphenyls by $p$ $\mathbf{O}_{2} \mathbf{N C}_{6} \mathbf{H}_{4} \mathbf{S C l}$. To a solution of 2-(1-alkynyl)biphenyl (0.30 mmol) in $\mathrm{CH}_{2} \mathrm{Cl}_{2}(3 \mathrm{~mL})$ was added $p-\mathrm{O}_{2} \mathrm{NC}_{6} \mathrm{H}_{4} \mathrm{SCl}$ (1.2 equiv) at room temperature. The reaction mixture was stirred for $0.5 \mathrm{~h}$ unless otherwise indicated. The reaction mixture was then diluted with diethyl ether $(50 \mathrm{~mL})$, washed with satd aq $\mathrm{NH}_{4} \mathrm{Cl}(25 \mathrm{~mL})$, dried $\left(\mathrm{MgSO}_{4}\right)$, and filtered. The solvent was evaporated under reduced pressure and the product was purified by chromatography on a silica gel column.<smiles>O=[N+]([O-])c1ccc(Sc2c(-c3ccccc3)c3ccccc3c3ccccc23)cc1</smiles>

9-(4-Nitrophenylsulfenyl)-10-phenylphenanthrene (4). Purification by flash chromatography (30:1 hexane/EtOAc) afforded $112 \mathrm{mg}(92 \%)$ of the product as a yellow

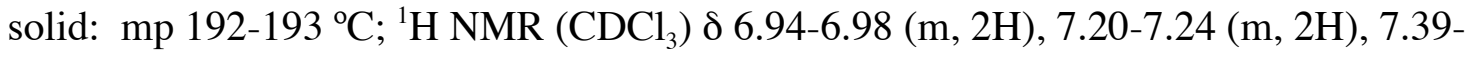
$7.47(\mathrm{~m}, 3 \mathrm{H}), 7.50-7.54(\mathrm{~m}, 2 \mathrm{H}), 7.71-7.64(\mathrm{~m}, 1 \mathrm{H}), 7.72-7.79(\mathrm{~m}, 2 \mathrm{H}), 7.93(\mathrm{dt}, J=9.3$, $2.1 \mathrm{~Hz}, 2 \mathrm{H}), 8.46(\mathrm{dd}, J=8.4,0.9 \mathrm{~Hz}, 1 \mathrm{H}), 8.83(\mathrm{~d}, J=8.4 \mathrm{~Hz}, 2 \mathrm{H}) ;{ }^{13} \mathrm{C} \mathrm{NMR}\left(\mathrm{CDCl}_{3}\right) \delta$ 123.0, 123.4, 124.1, 125.0, 125.9, 127.3, 127.4, 127.8, 128.1, 128.3, 128.4, 128.6, 129.2, 129.4, 131.3, 131.6, 131.7, 132.3, 139.9, 145.1, 148.0, 149.2; IR (neat, $\mathrm{cm}^{-1}$ ) 3066, 3024, 2834, 1610; HRMS Calcd for $\mathrm{C}_{26} \mathrm{H}_{7} \mathrm{NO}_{2} \mathrm{~S}$ : 407.0980. Found: 407.0989. 


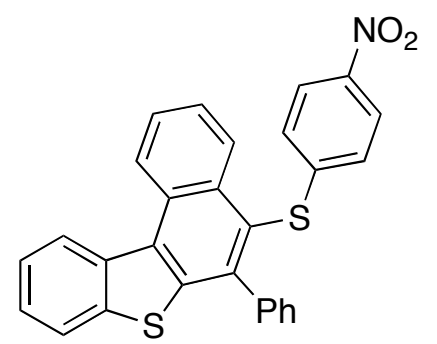

42

5-(4-Nitrophenylthio)-6-phenylbenzo[b]naphtha[1,2- $d]$ thiophene (42). Purification by flash chromatography (9:1 hexane/EtOAc) afforded $101 \mathrm{mg}(91 \%)$ of the product as a yellow solid: $\mathrm{mp}>215{ }^{\circ} \mathrm{C}$ (decomposed); ${ }^{1} \mathrm{H} \mathrm{NMR}\left(\mathrm{CDCl}_{3}\right) \delta$ 6.93-6.97 (m, 2H), 7.34$7.38(\mathrm{~m}, 2 \mathrm{H}), 7.43-7.57(\mathrm{~m}, 4 \mathrm{H}), 7.61-7.68(\mathrm{~m}, 2 \mathrm{H}), 7.79-7.85(\mathrm{~m}, 1 \mathrm{H})$, 7.92-7.95 (m, $3 \mathrm{H}), 8.63(\mathrm{dd}, J=8.7,0.9 \mathrm{~Hz}, 1 \mathrm{H}), 8.94(\mathrm{~d}, J=8.4 \mathrm{~Hz}, 1 \mathrm{H}), 9.17(\mathrm{~d}, J=5.4 \mathrm{~Hz}, 1 \mathrm{H}) ;{ }^{13} \mathrm{C}$ $\operatorname{NMR}\left(\mathrm{CDCl}_{3}\right) \delta 123.4,124.2,125.5,125.6,126.0,126.5,126.9,128.03,128.04,128.7$, 129.0, 129.0, 131.2, 131.7, 132.8, 136.6, 139.9, 141.3, 141.4, 143.3, 145.2, 149.1 (two $\mathrm{sp}^{2}$ carbons missing due to overlap); IR (neat, $\mathrm{cm}^{-1}$ ) 3060, 2924, 1579, 1513, 1336; HRMS Calcd for $\mathrm{C}_{28} \mathrm{H}_{17} \mathrm{NO}_{2} \mathrm{~S}_{2}$ : 463.0701 Found: 463.0713 .<smiles>CCOC(=O)/C=C/c1c(-c2ccccc2)c2ccccc2c2ccccc12</smiles>

57

Ethyl (2E)-3-(10-phenyl-9-phenanthryl)acrylate (57). To a solution of 9-iodo-10phenylphenanthrene $(0.20 \mathrm{mmol})$ and ethyl acrylate $(1.0 \mathrm{mmol}, 5.0$ equiv) in DMF $(0.8$ $\mathrm{mL})$ were added $\mathrm{Pd}(\mathrm{OAc})_{2}(2.2 \mathrm{mg}, 5 \mathrm{~mol} \%), n-\mathrm{Bu}_{4} \mathrm{NCl}(0.20 \mathrm{mmol}, 1$ equiv $)$ and $\mathrm{NaHCO}_{3}(0.5 \mathrm{mmol}, 2.5$ equiv $)$. The resulting mixture was heated under a $\mathrm{N}_{2}$ atmosphere at $100{ }^{\circ} \mathrm{C}$ for $3 \mathrm{~d}$. The mixture was cooled to room temperature and diluted with $70 \mathrm{~mL}$ 
of ether, washed with $25 \mathrm{~mL}$ of satd aq $\mathrm{NaCl}$, dried $\left(\mathrm{MgSO}_{4}\right)$ and filtered. The solvent was evaporated under reduced pressure. The residue was chromatographed using 7:1 hexane/EtOAc to afford $69.0 \mathrm{mg}(98 \%)$ of the product as a yellow solid: $\mathrm{mp} 135-136{ }^{\circ} \mathrm{C}$; ${ }^{1} \mathrm{H}$ NMR $\left(\mathrm{CDCl}_{3}\right) \delta 1.28(\mathrm{t}, J=7.1 \mathrm{~Hz}, 3 \mathrm{H}), 4.20(\mathrm{q}, J=6.9 \mathrm{~Hz}, 2 \mathrm{H}), 6.02(\mathrm{~d}, J=16.2$ $\mathrm{Hz}, 1 \mathrm{H}), 7.26-7.31(\mathrm{~m}, 2 \mathrm{H}), 7.44-7.55(\mathrm{~m}, 5 \mathrm{H}), 7.62-7.75(\mathrm{~m}, 3 \mathrm{H}), 7.88(\mathrm{~d}, J=16.2 \mathrm{~Hz}$, $1 \mathrm{H}), 8.23(\mathrm{dd}, J=1.2,8.1 \mathrm{~Hz}, 1 \mathrm{H}), 8.73-8.81(\mathrm{~m}, 2 \mathrm{H}) ;{ }^{13} \mathrm{C} \mathrm{NMR}\left(\mathrm{CDCl}_{3}\right) \delta 14.5,60.7$, $122.7,123.2,126.1,126.5,127.0,127.1,127.31,127.34,127.8,128.3,128.6,129.9$, 130.2, 130.4, 130.6, 130.9, 131.7, 138.3, 139.0, 143.5, 166.6; IR (neat, $\mathrm{cm}^{-1}$ ) 3066, 2982, 1712, 1642, 1488; HRMS Calcd for $\mathrm{C}_{25} \mathrm{H}_{20} \mathrm{O}_{2}$ : 352.1463. Found: 352.1469 .

\section{References}

1. Yao, T. Campo, M. A.; Larock, R. C. Org. Lett. 2004, 6, 2677.

2. Campo, M. A.; Larock, R. C. Org. Lett. 2000, $2,3675$. 

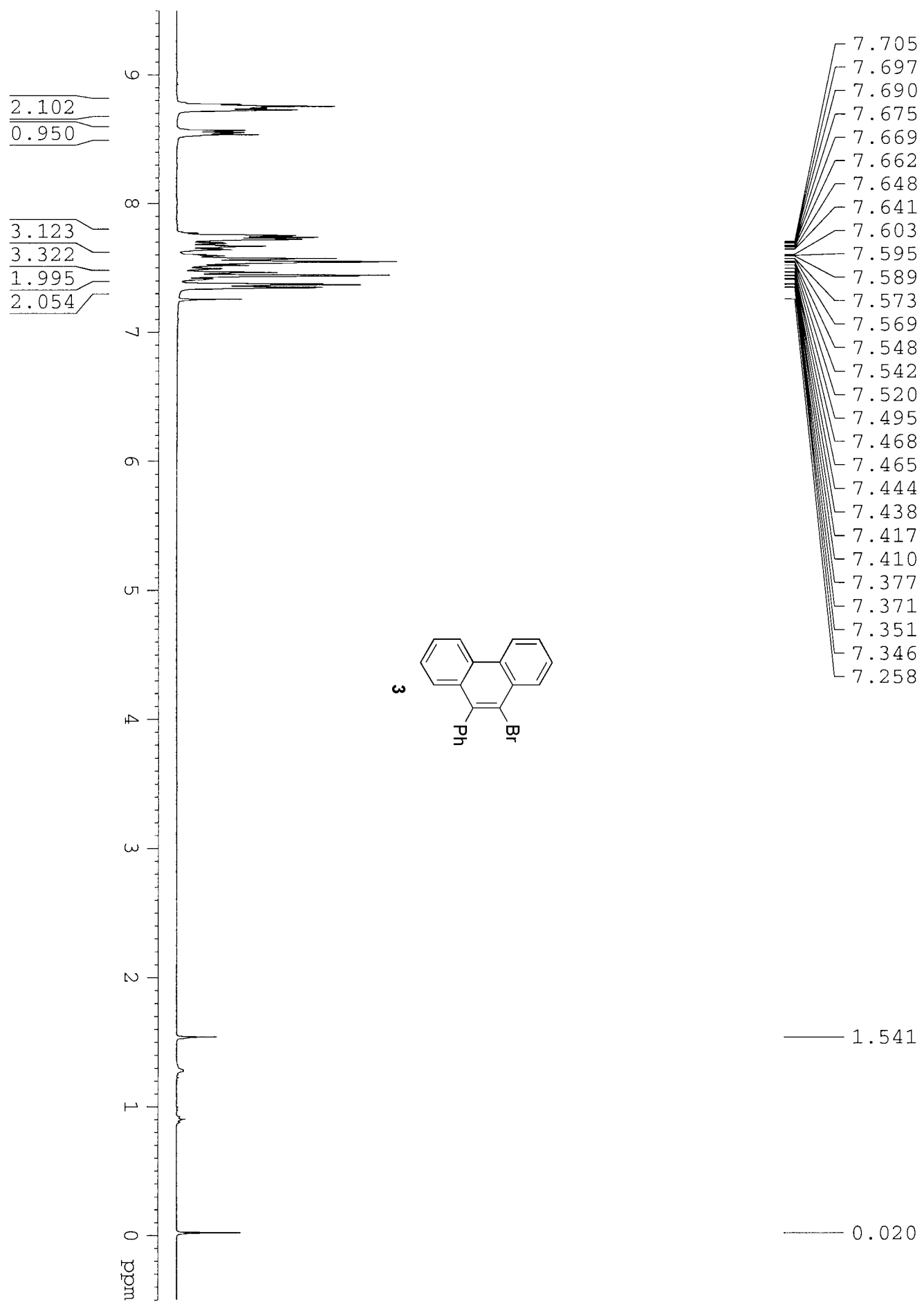

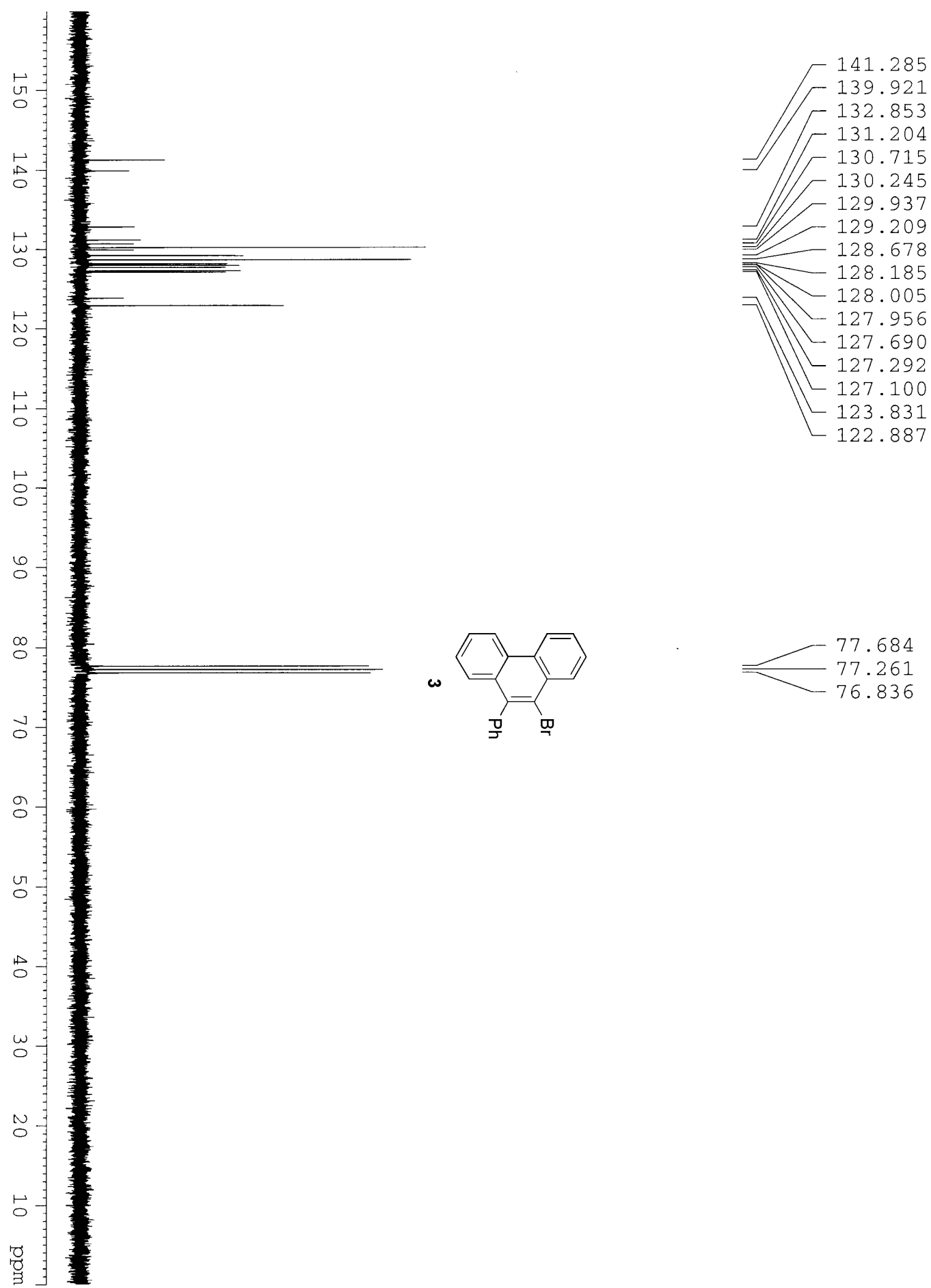

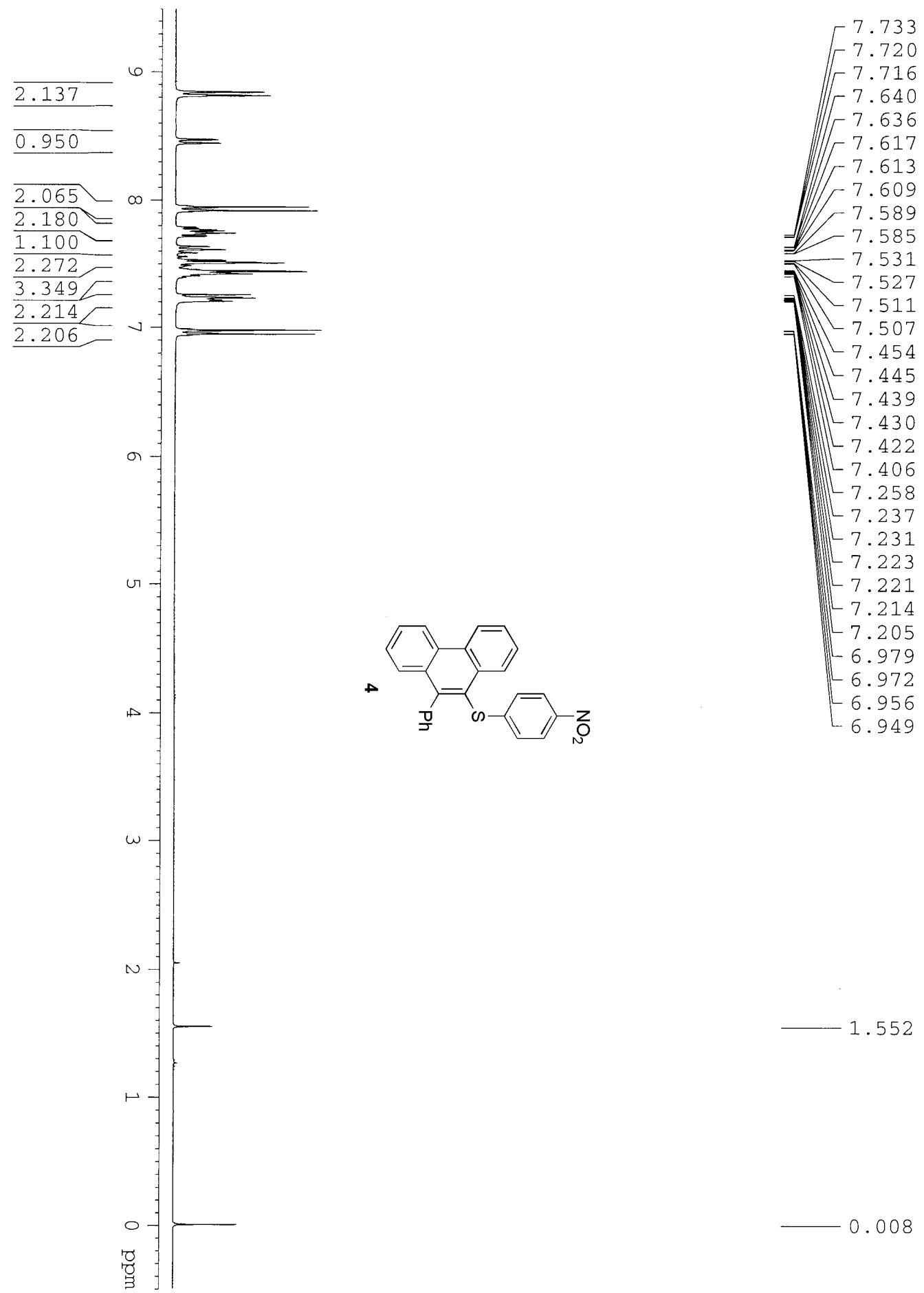

$-0.008$ 

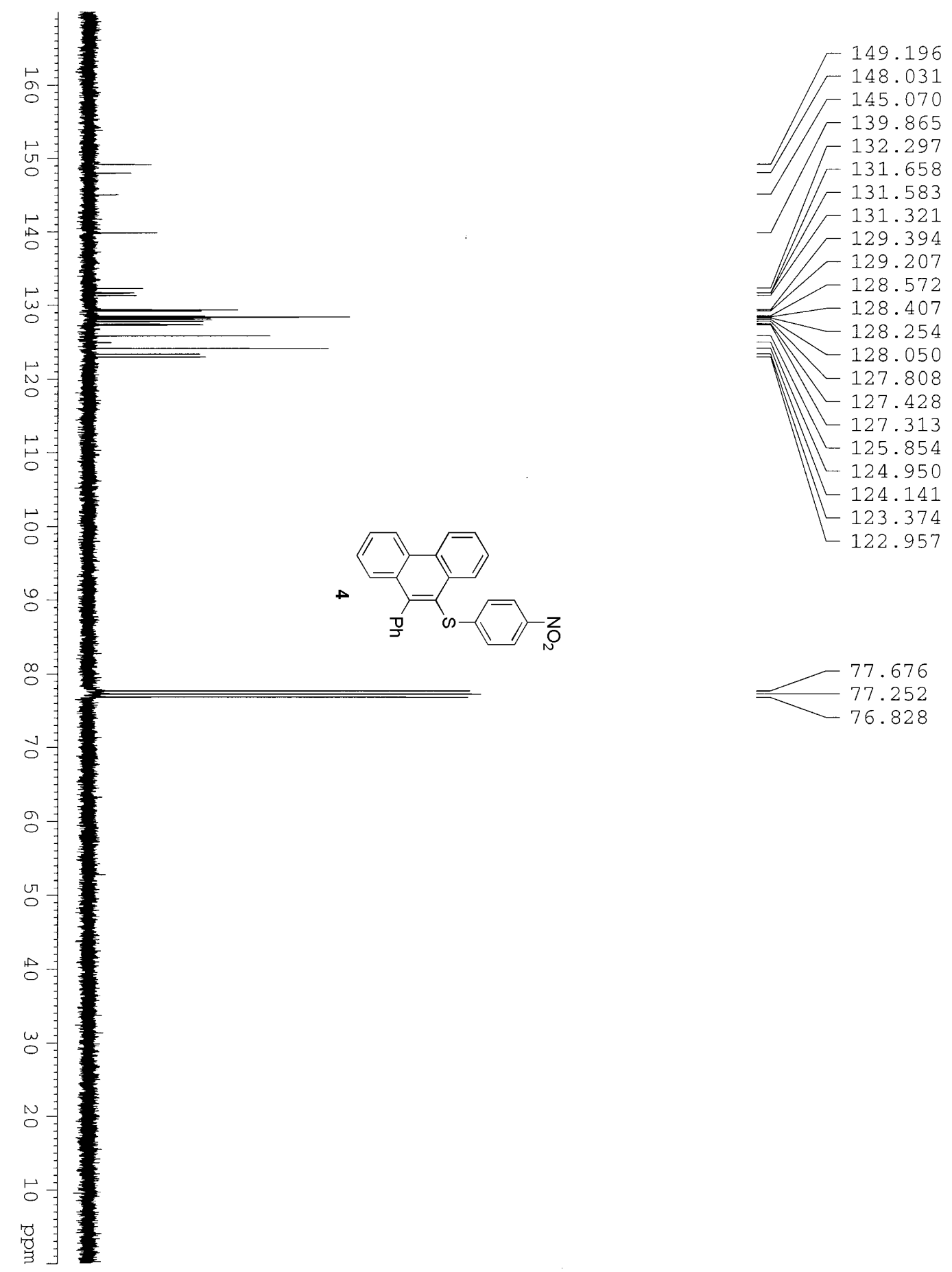

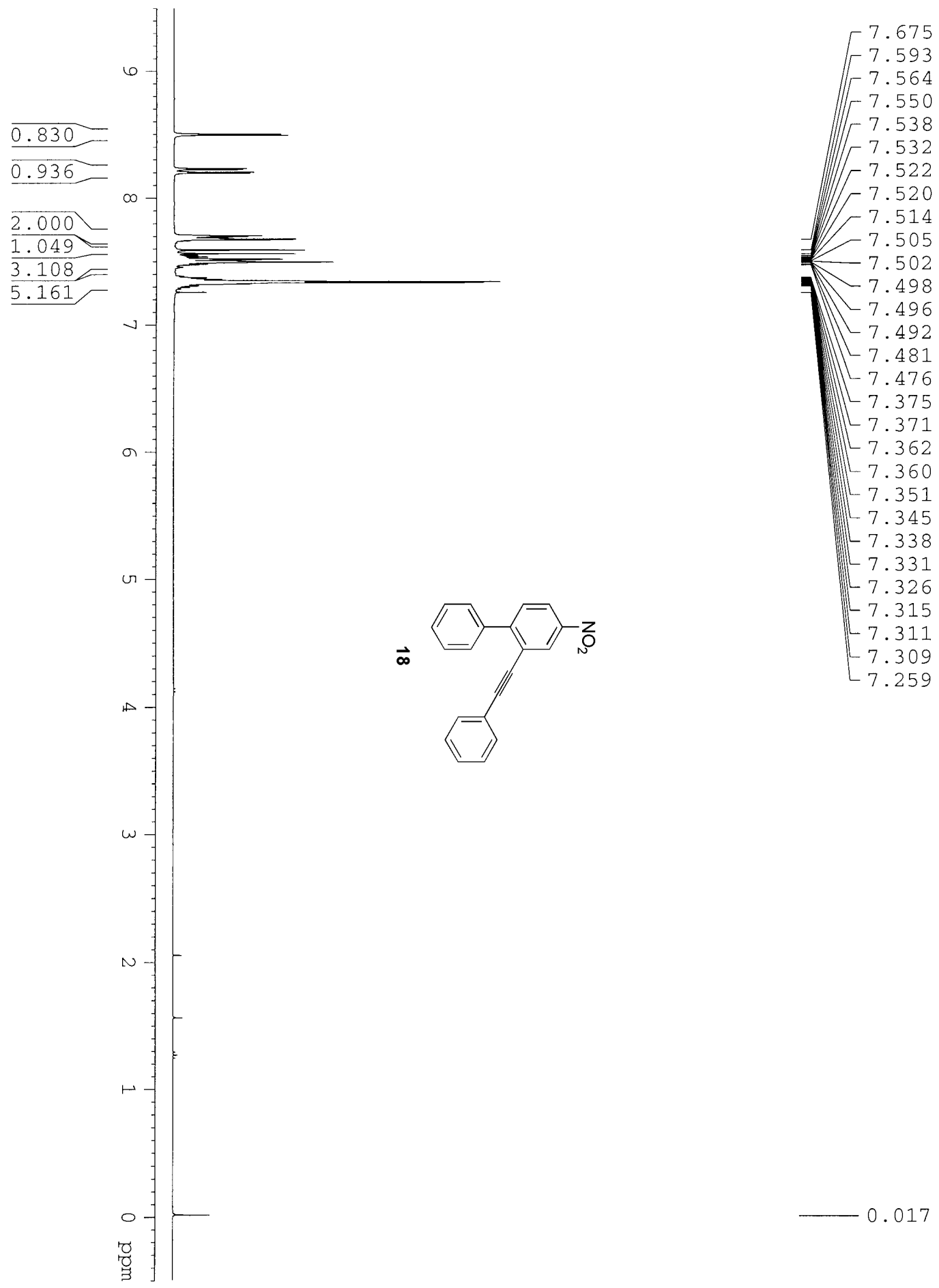

$-0.017$ 

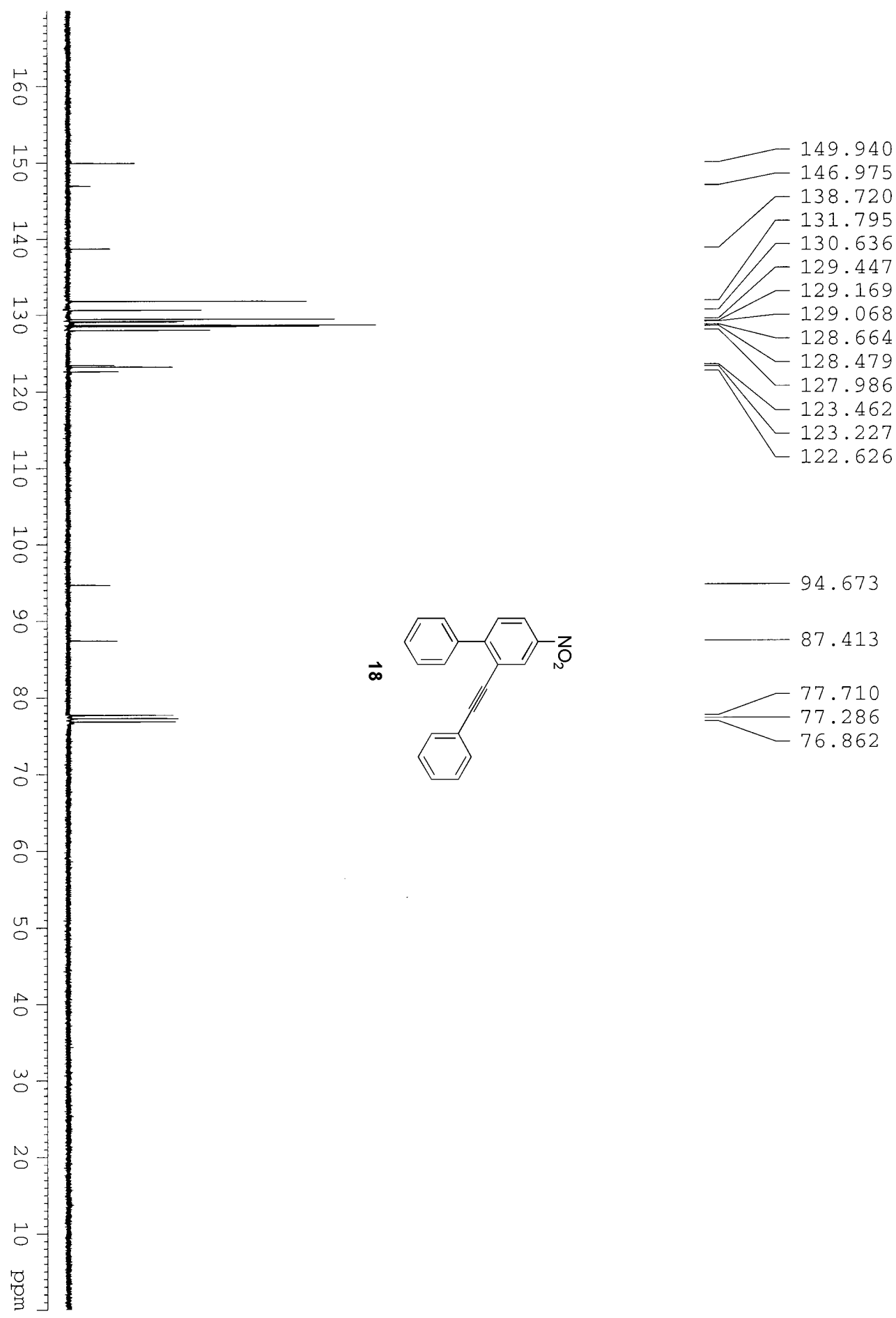

87.413

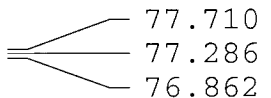



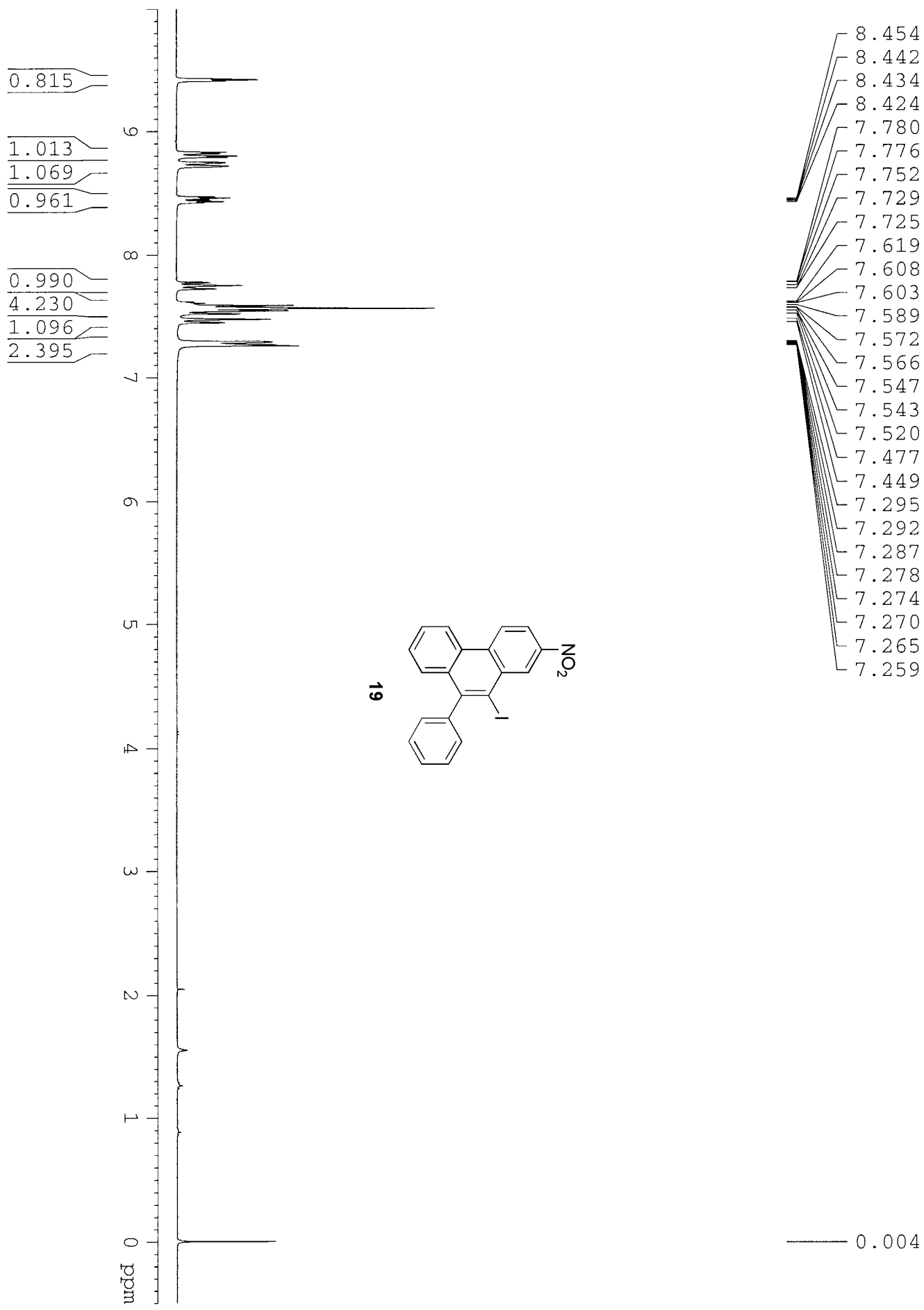

$-0.004$ 

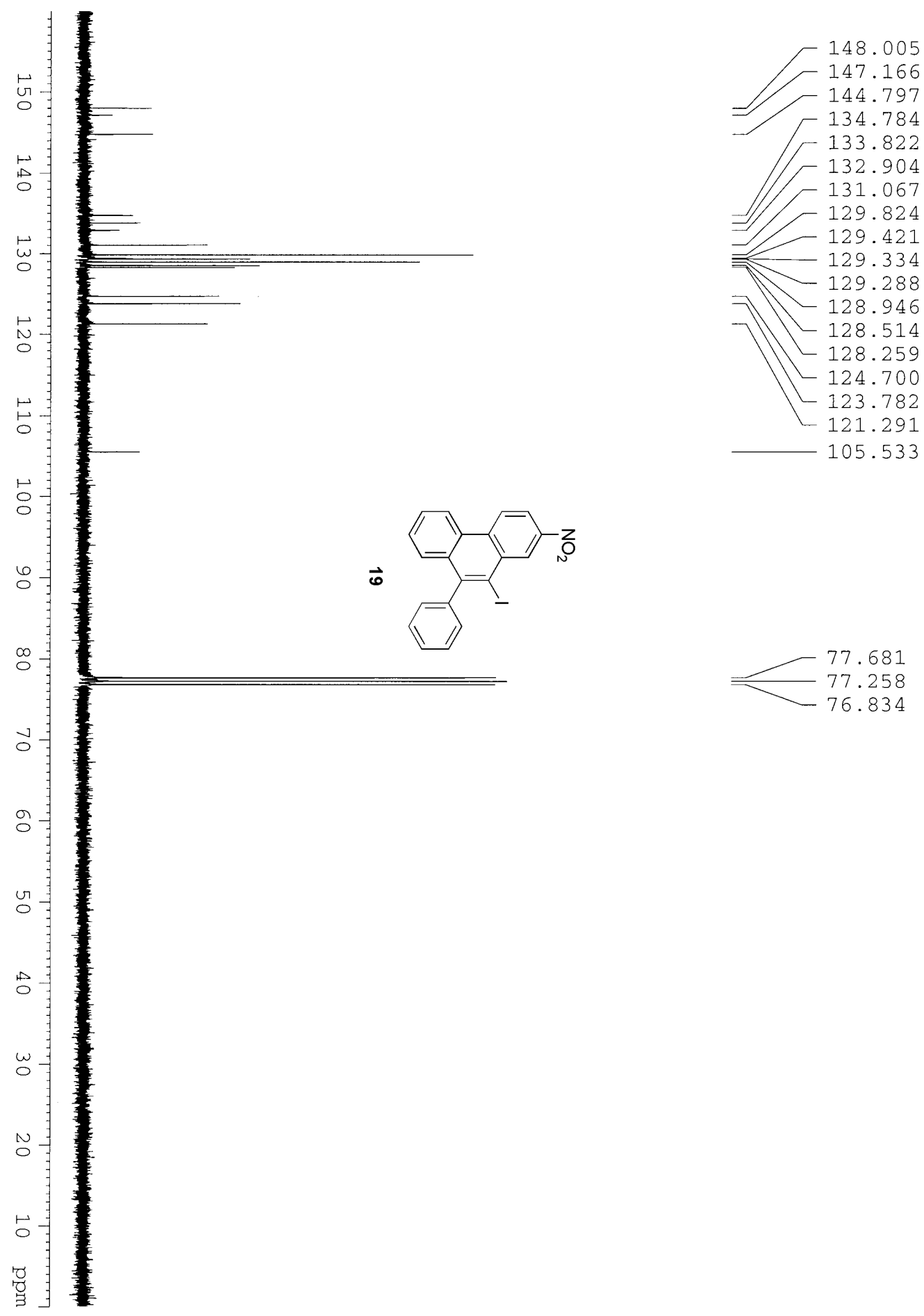

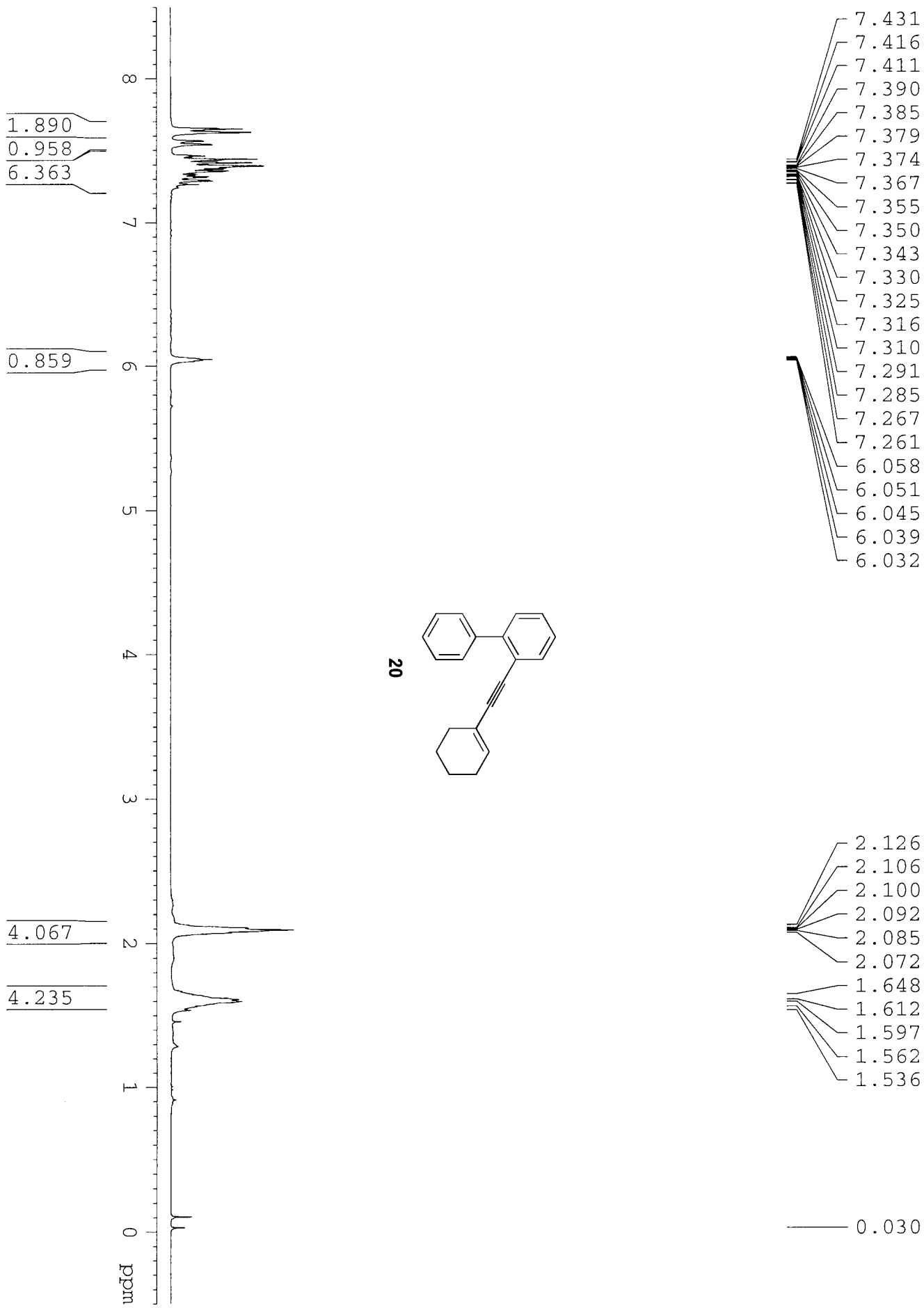

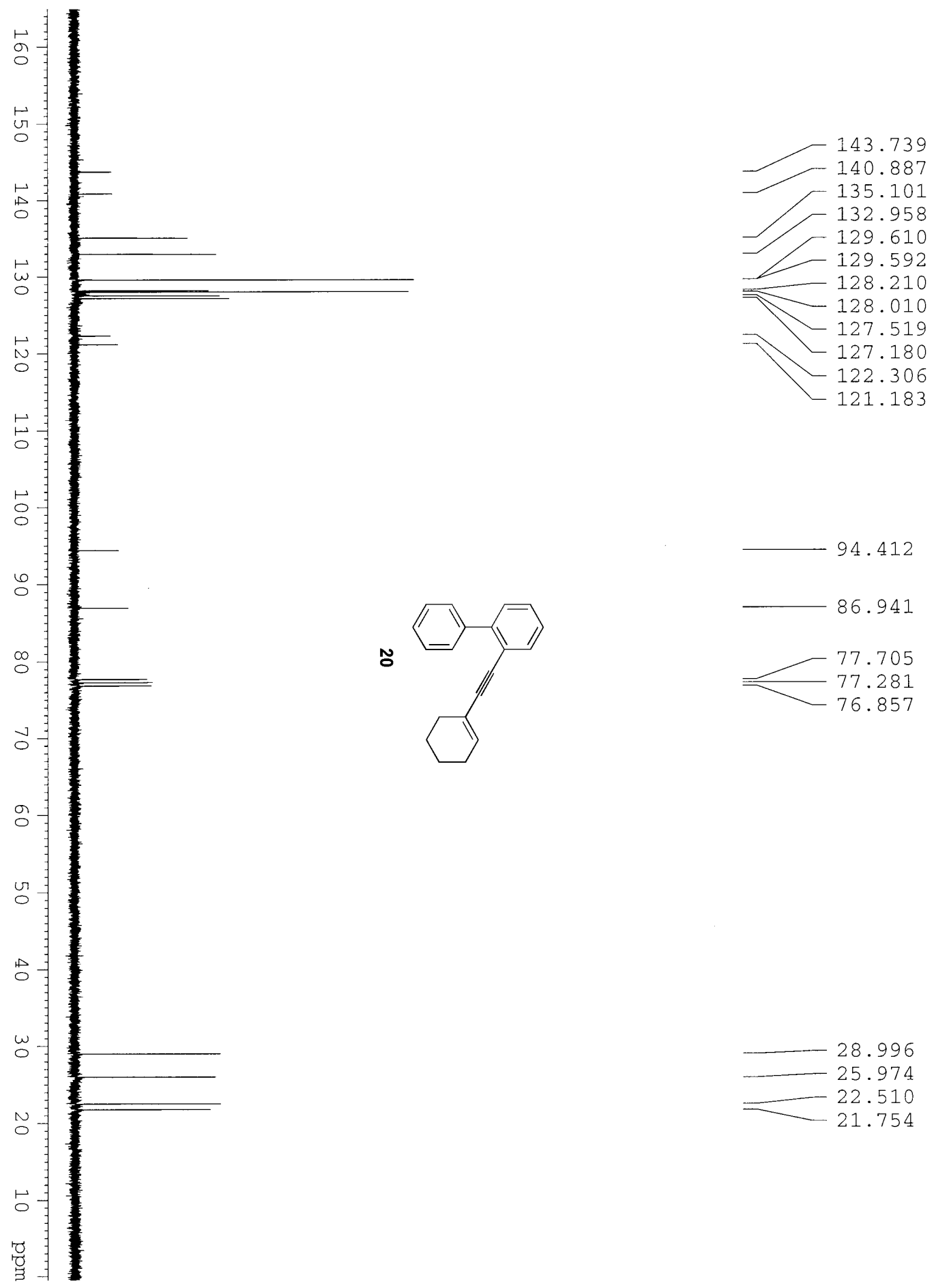

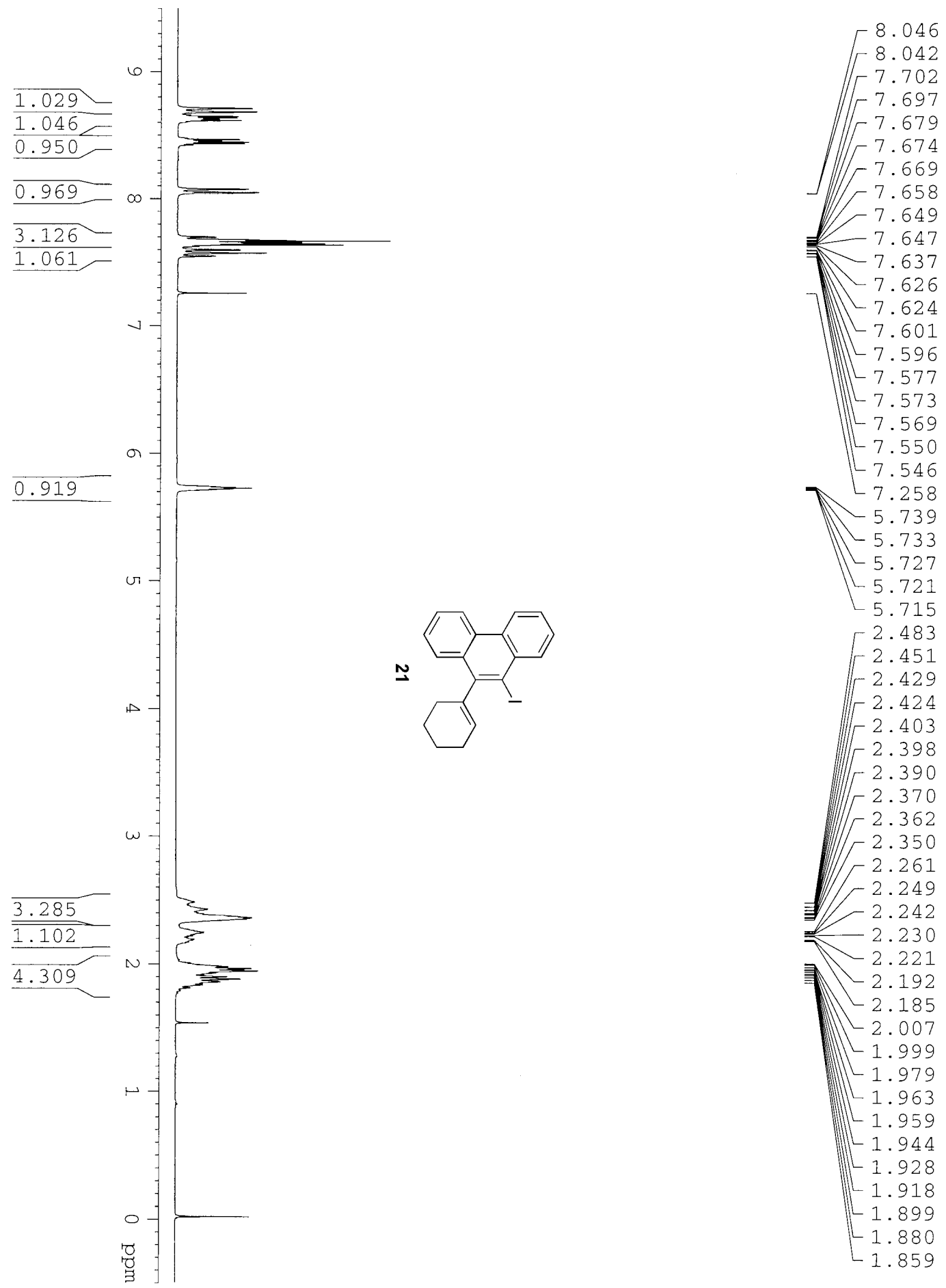

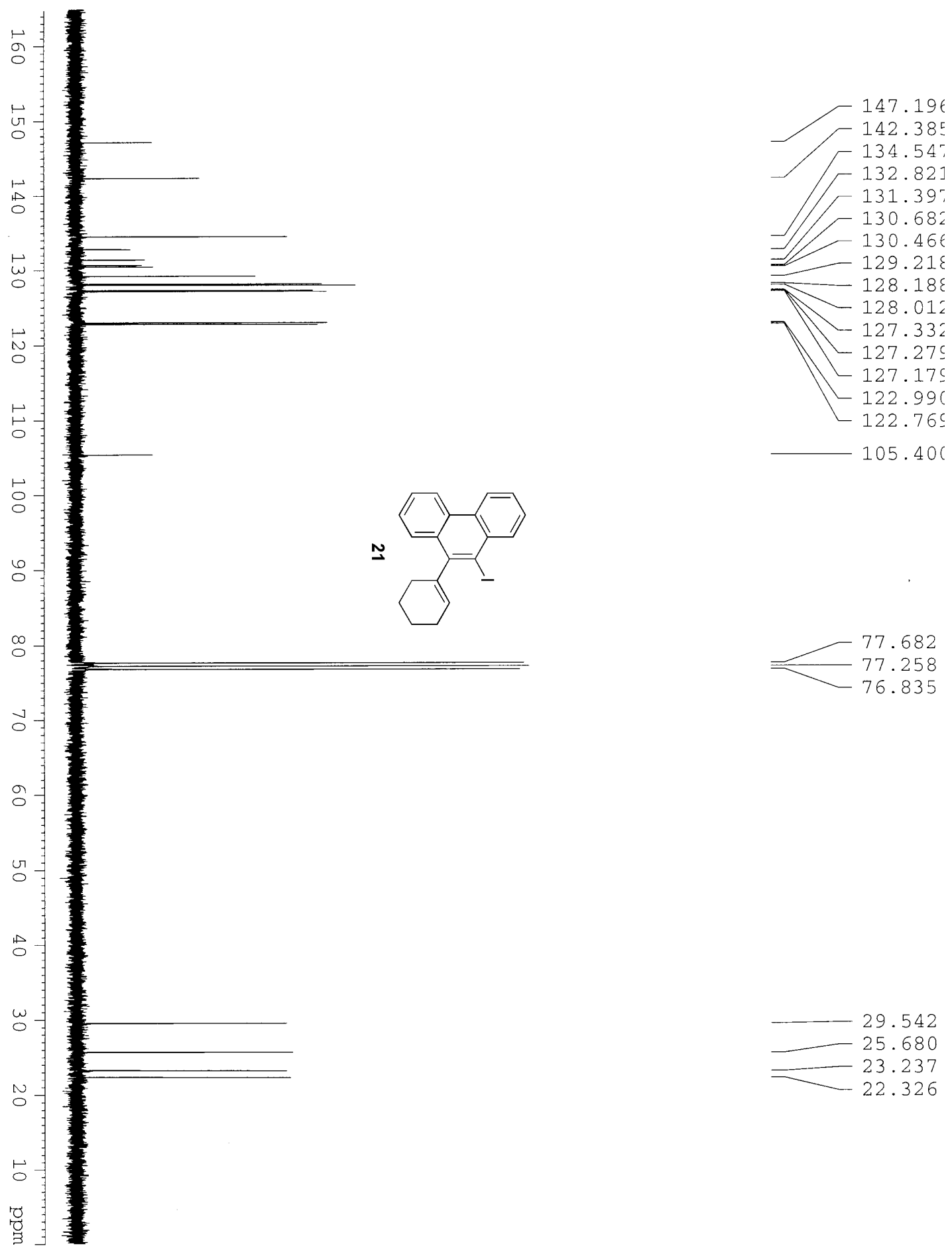


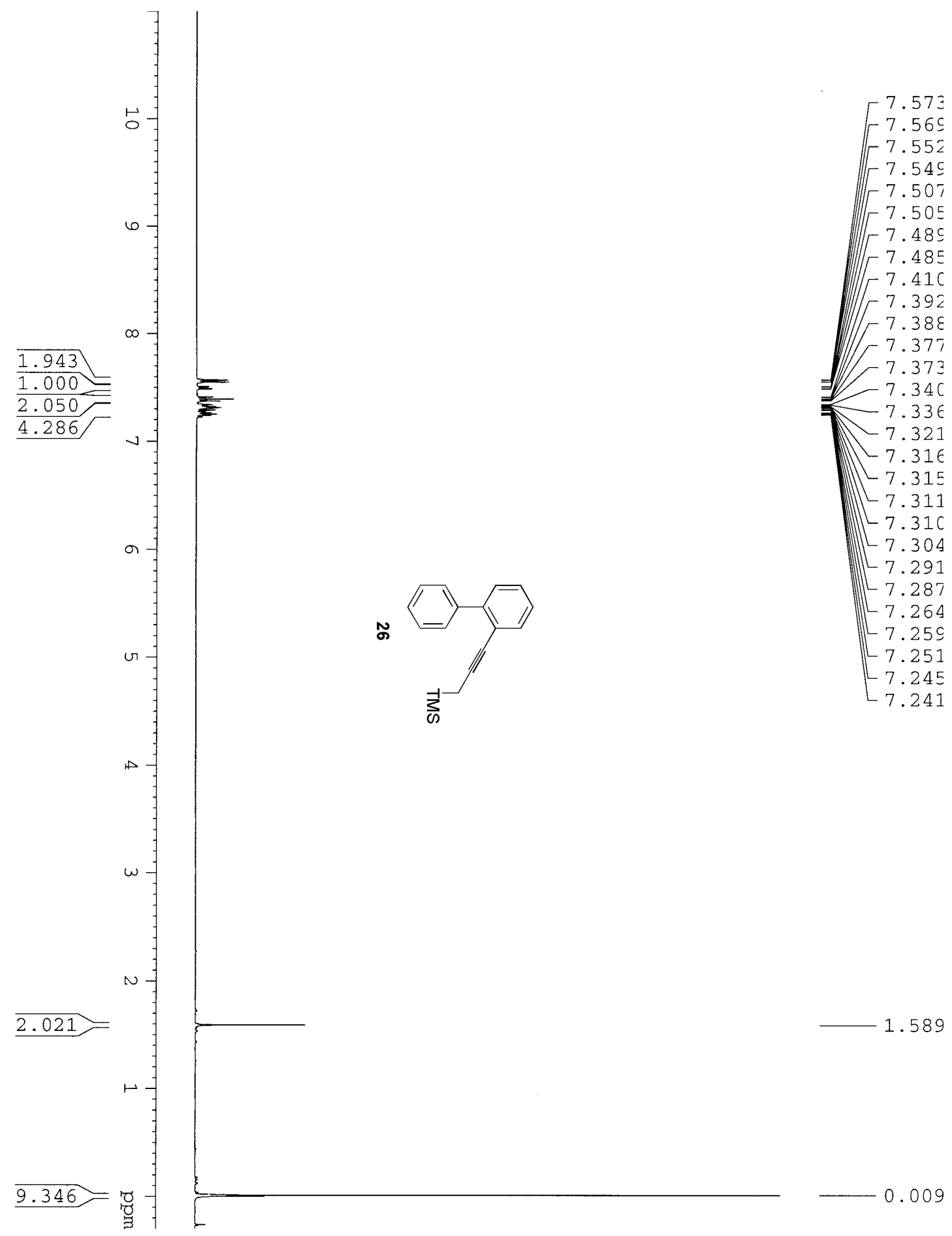



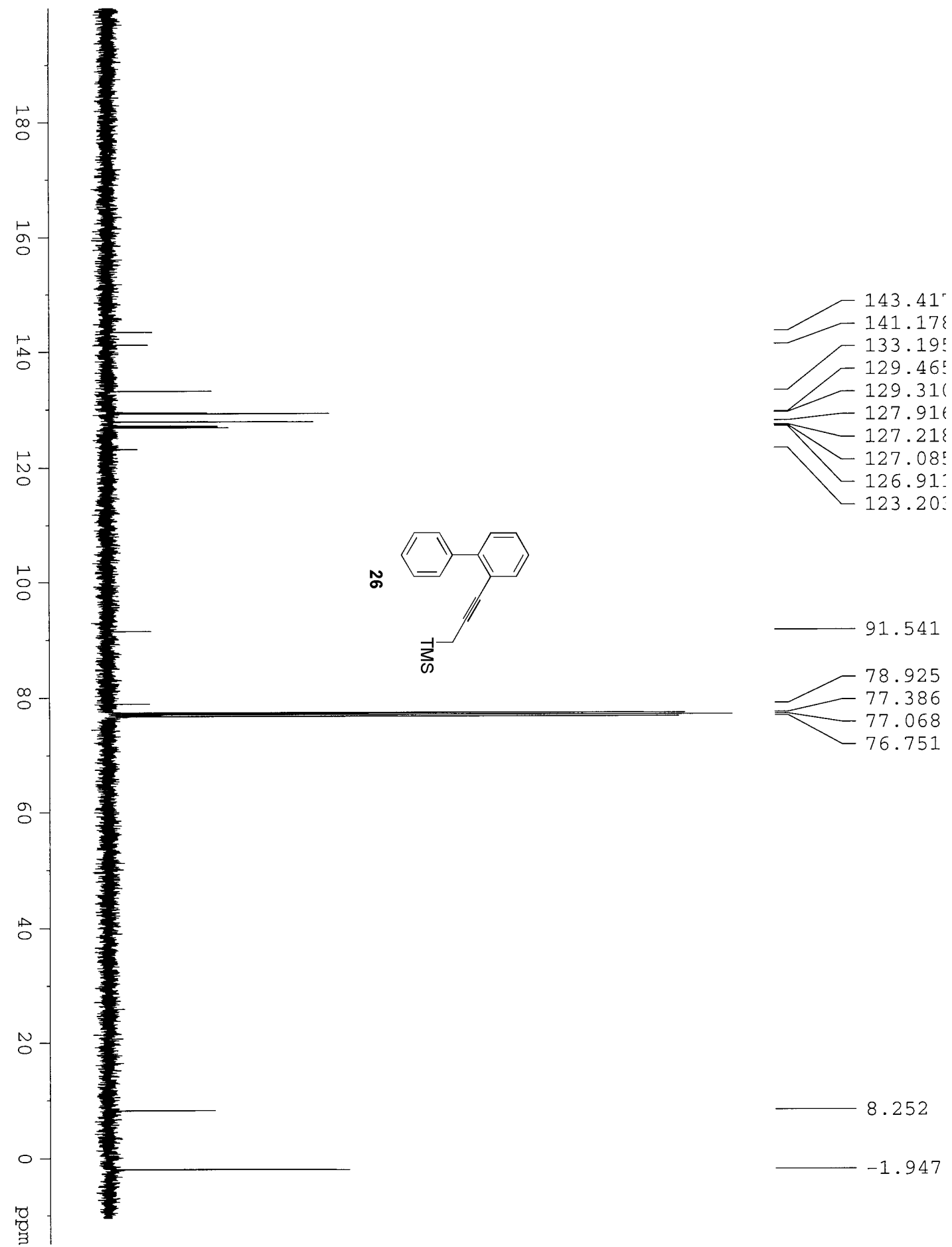

8.252

$-1.947$ 

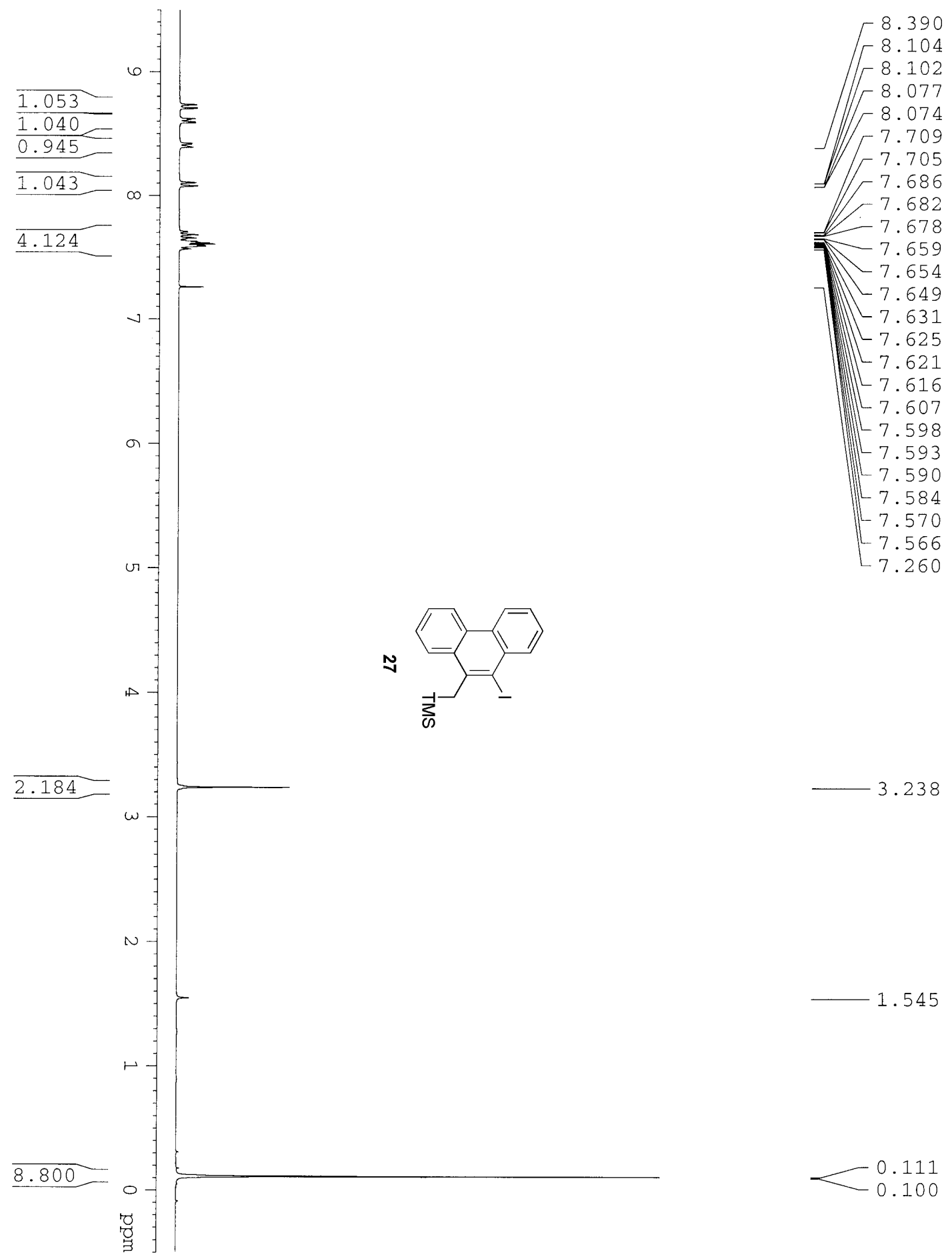

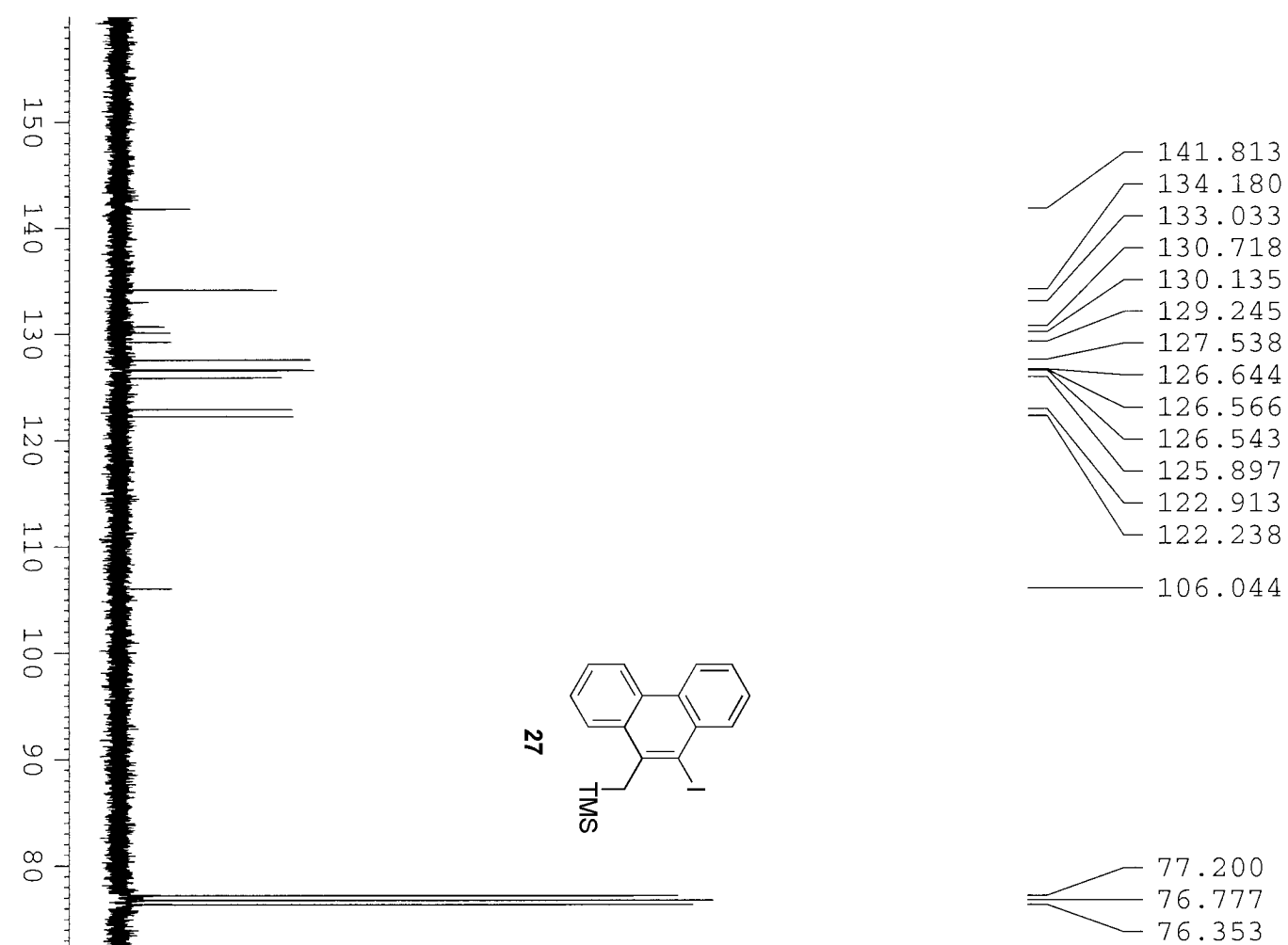

31.102

w

N

5

ס

o

G

$\stackrel{\circ}{0}$

w

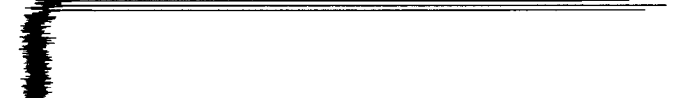

0.007 

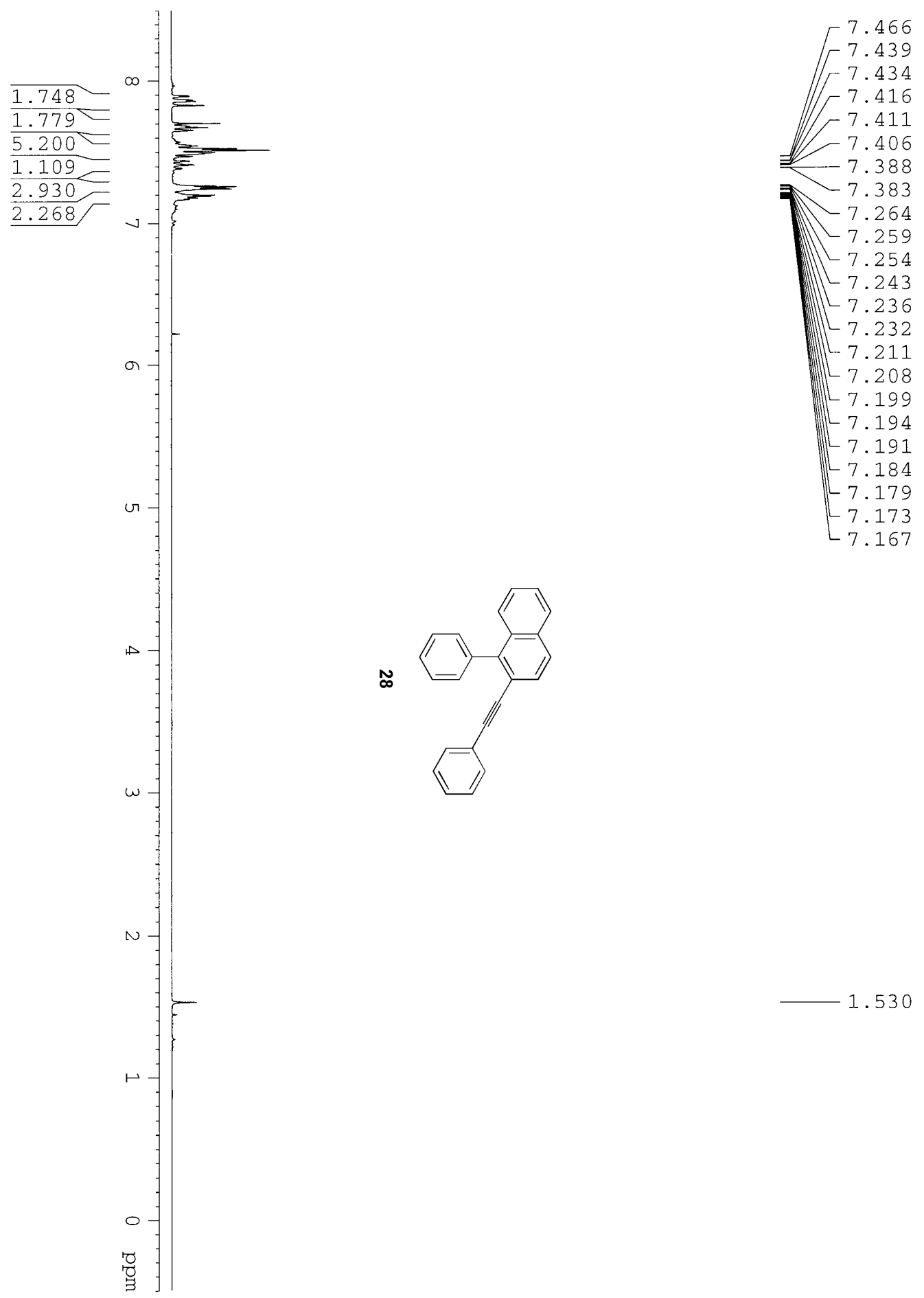

1.530 


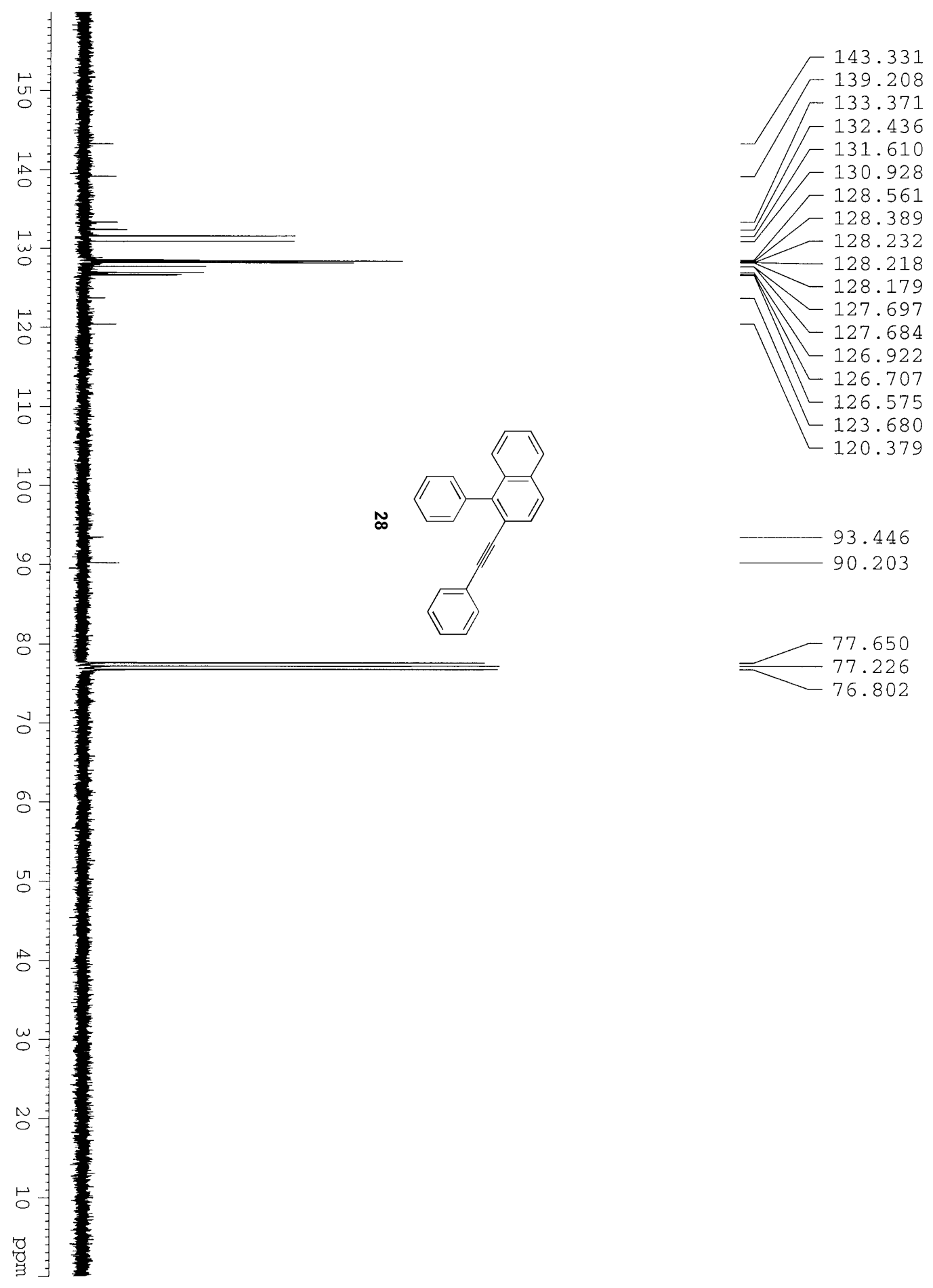




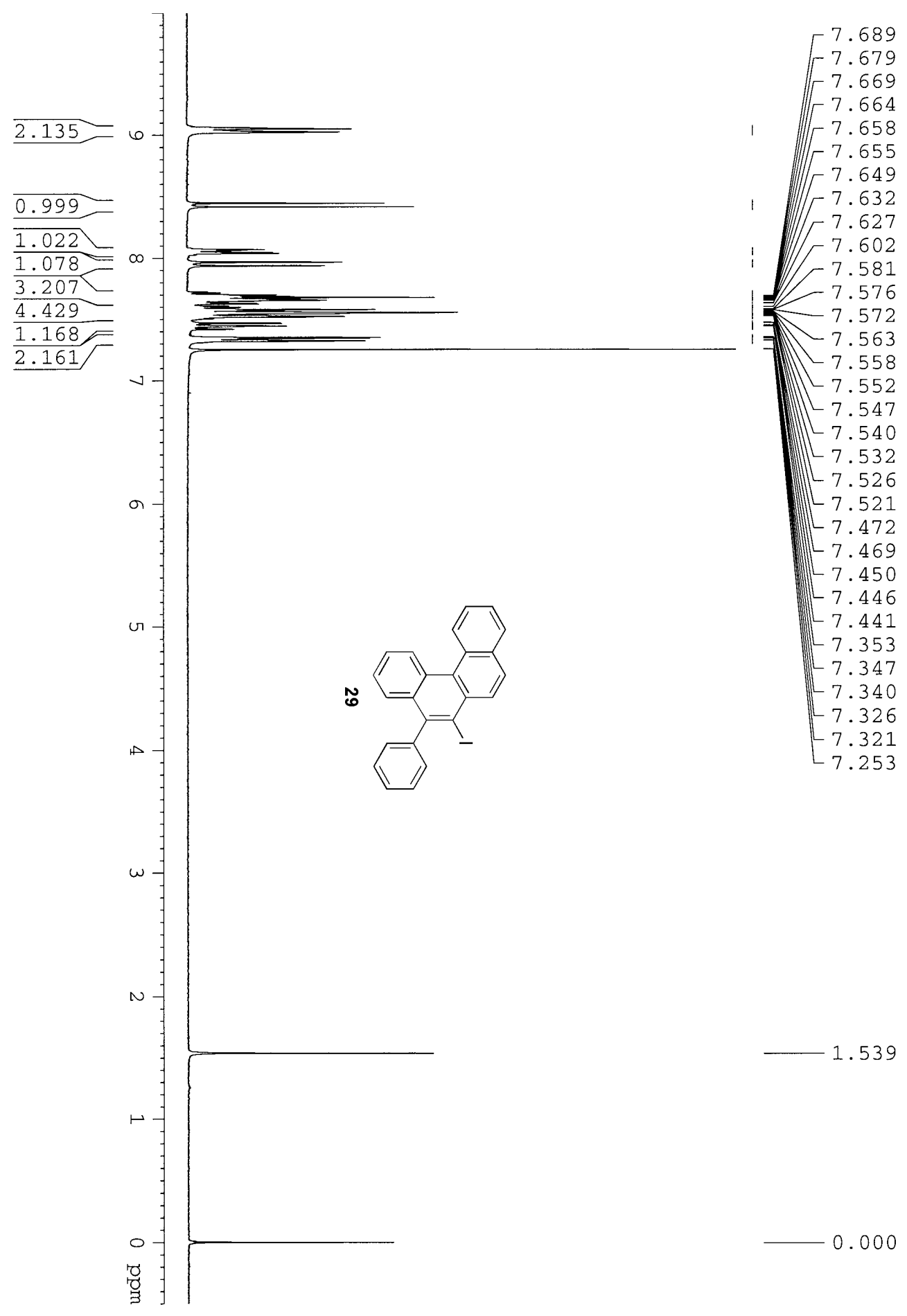




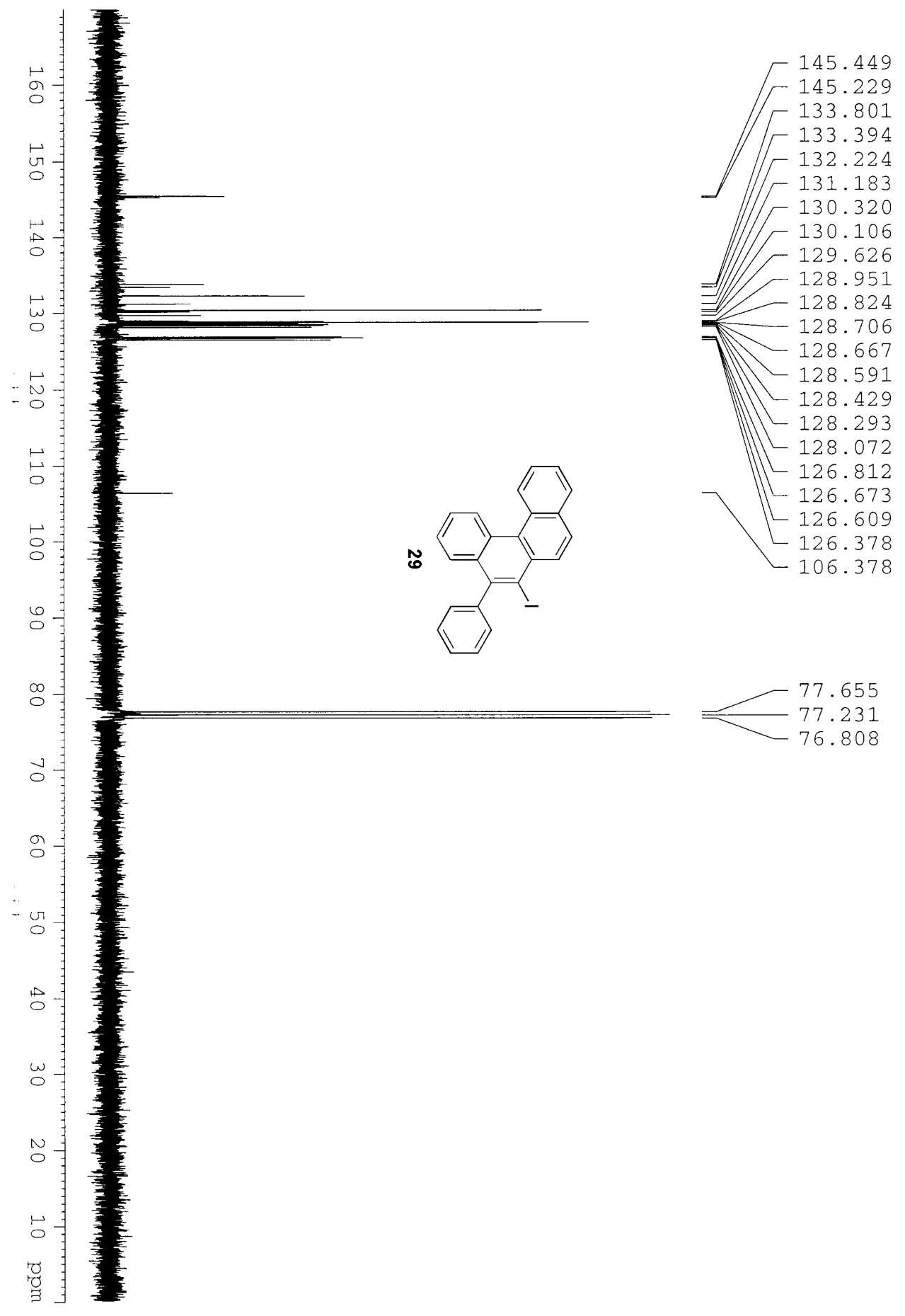




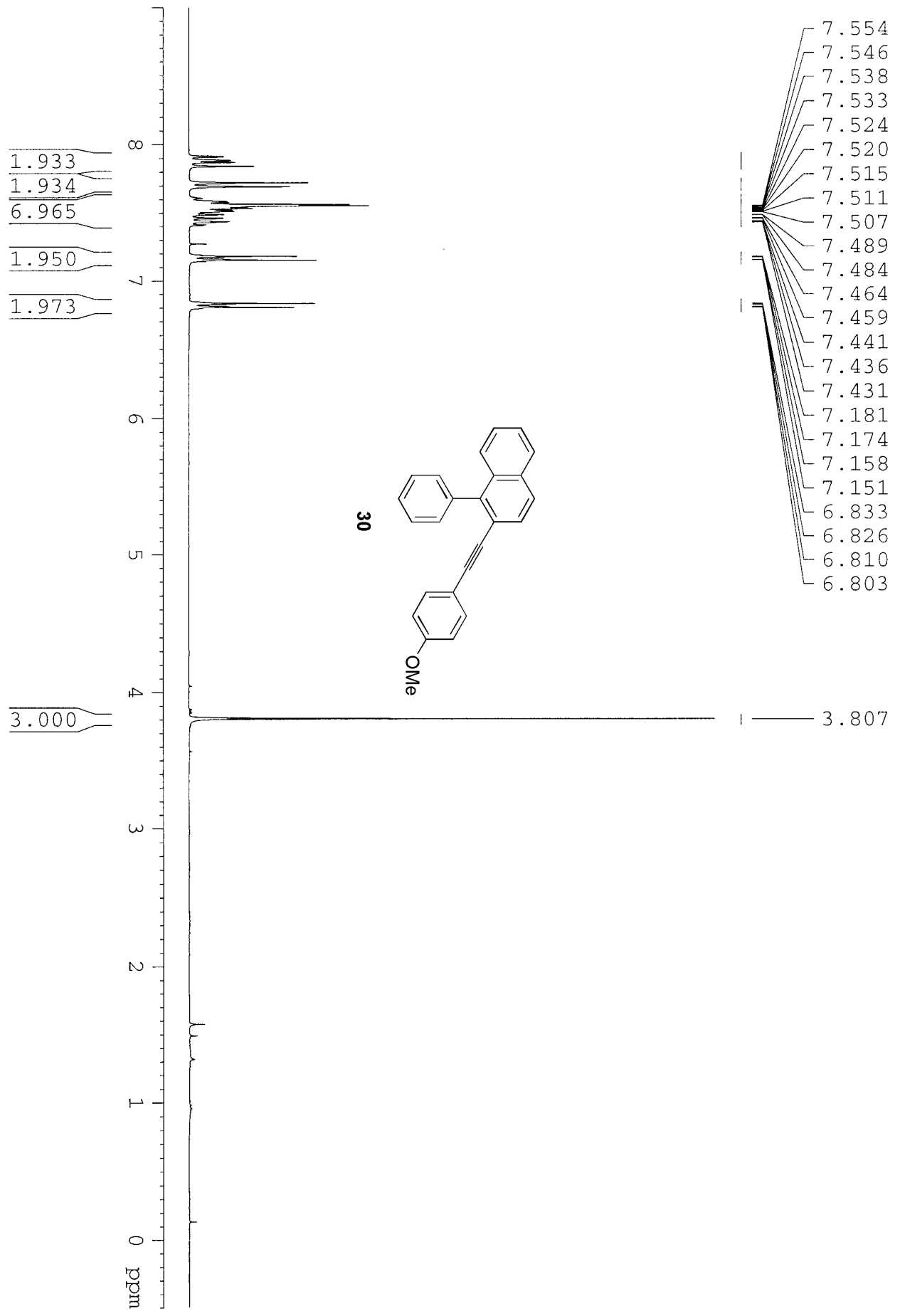



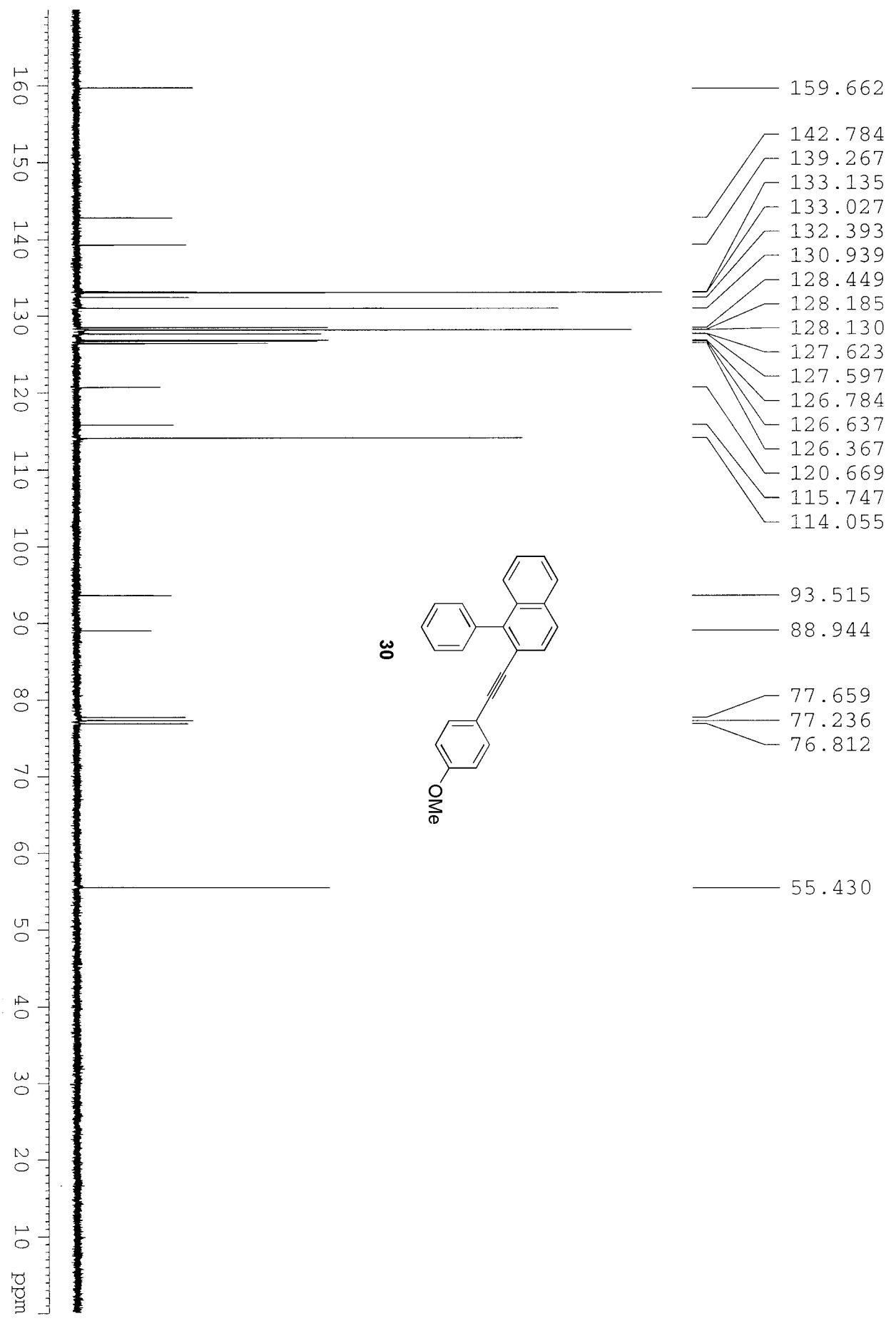

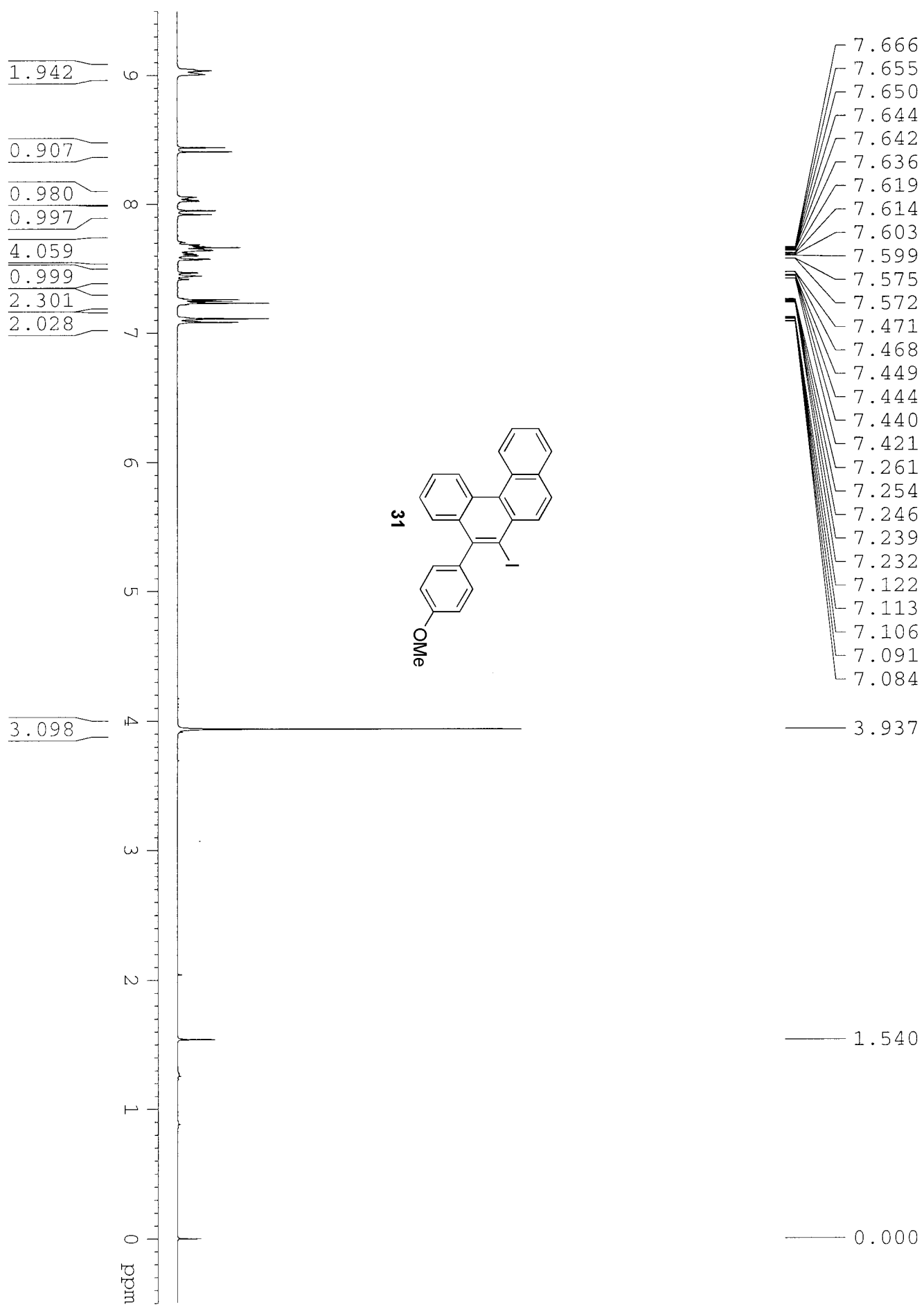

.540

0.000 


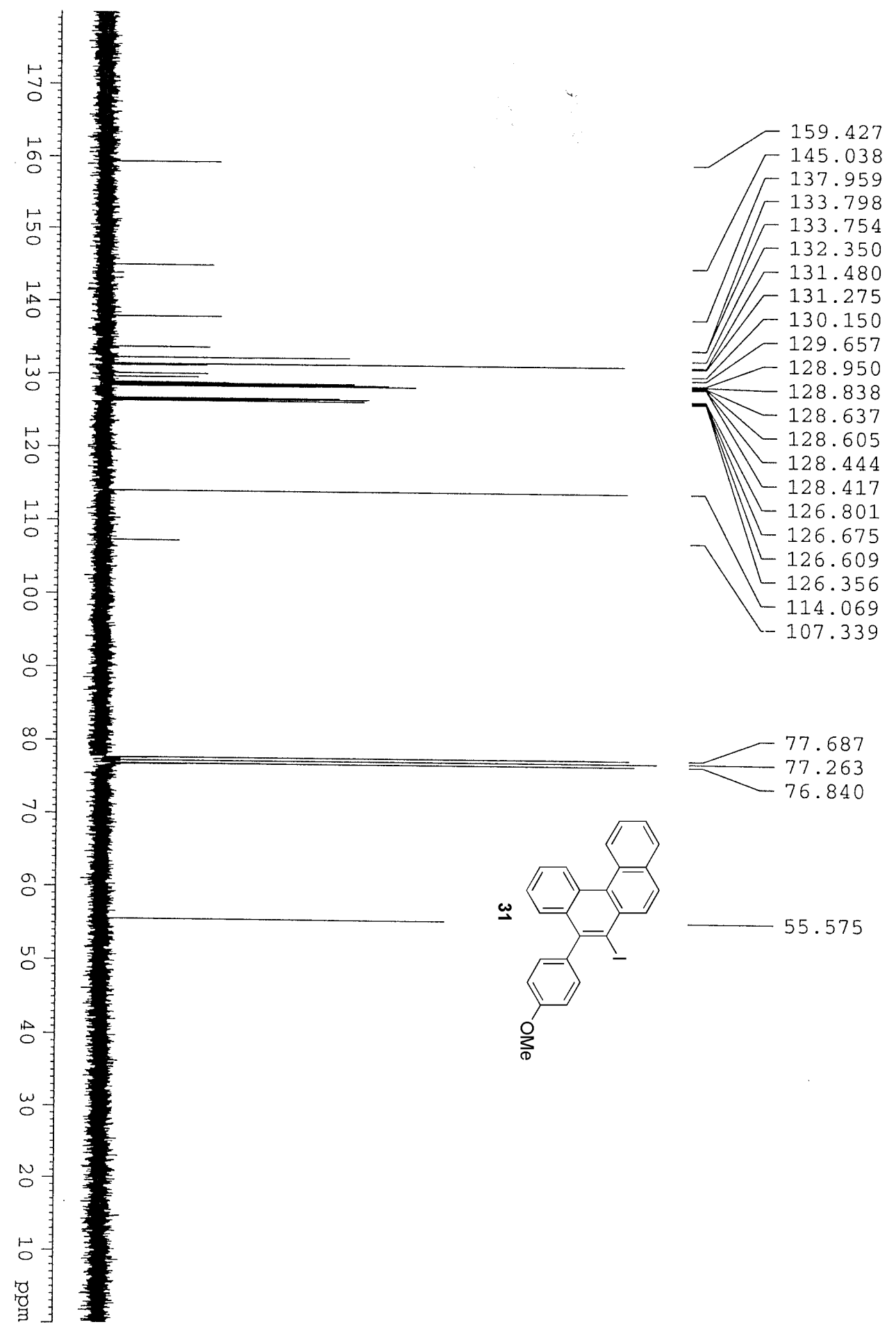



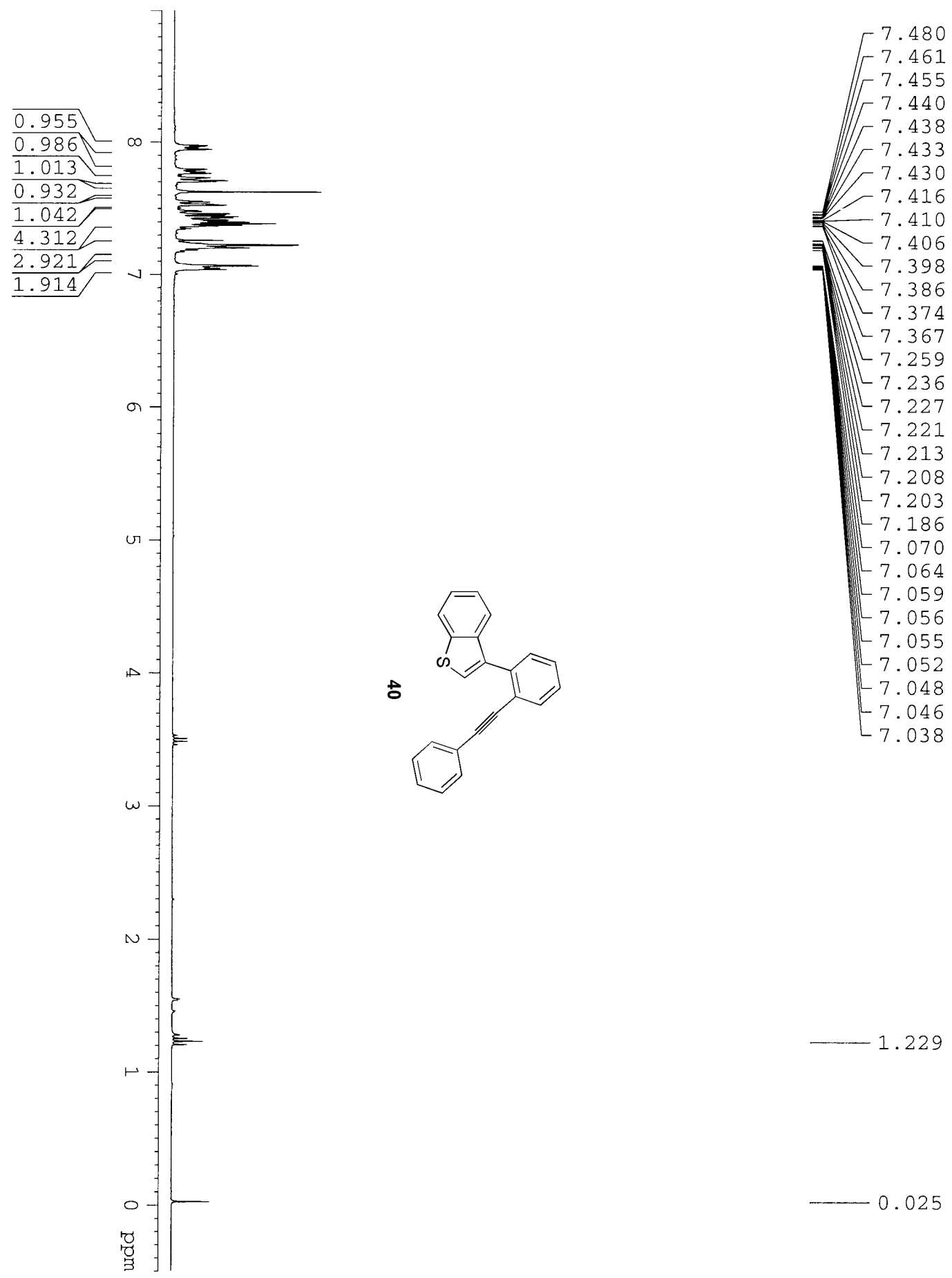

$-1.229$

$-0.025$

S41 

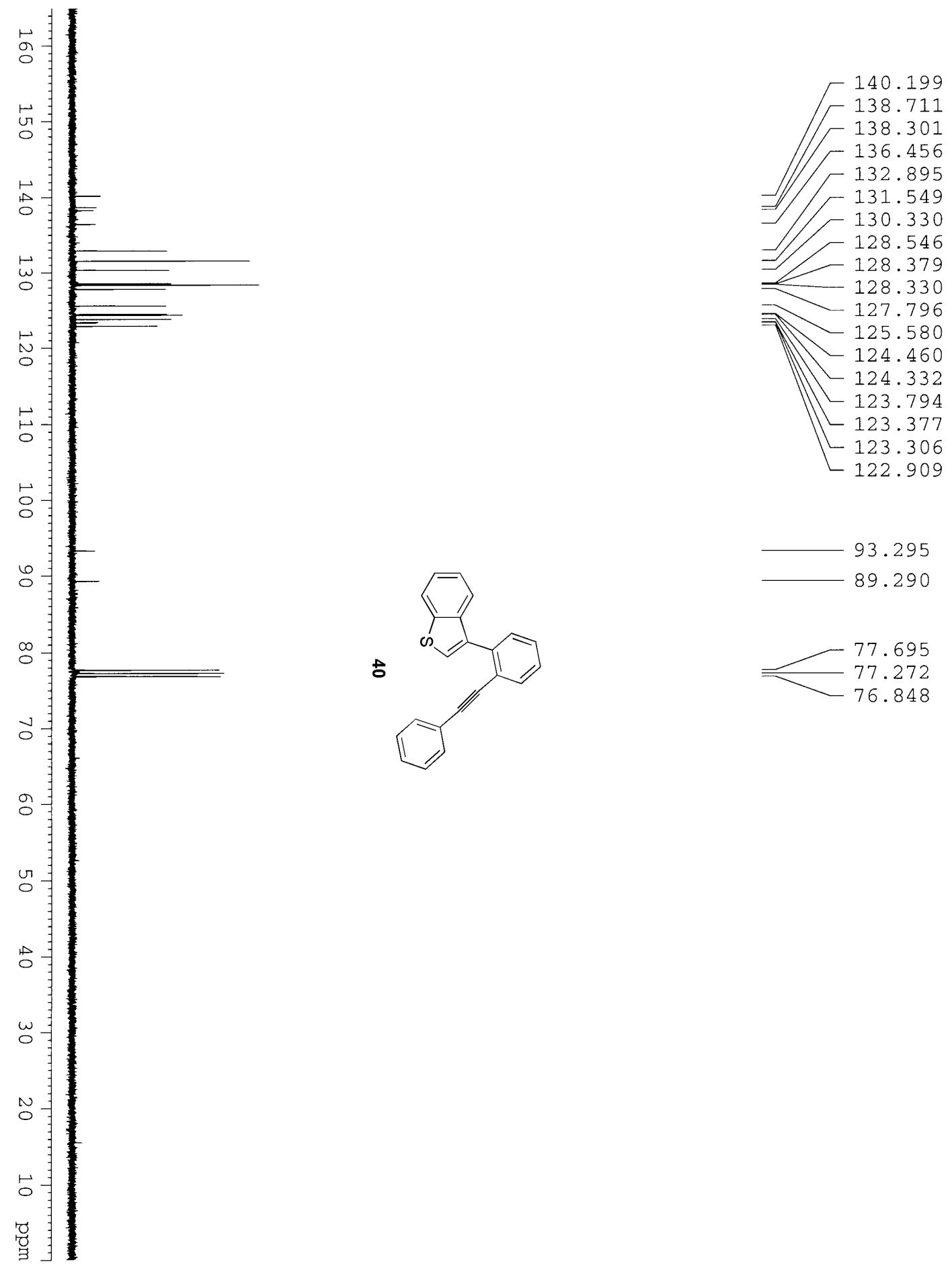

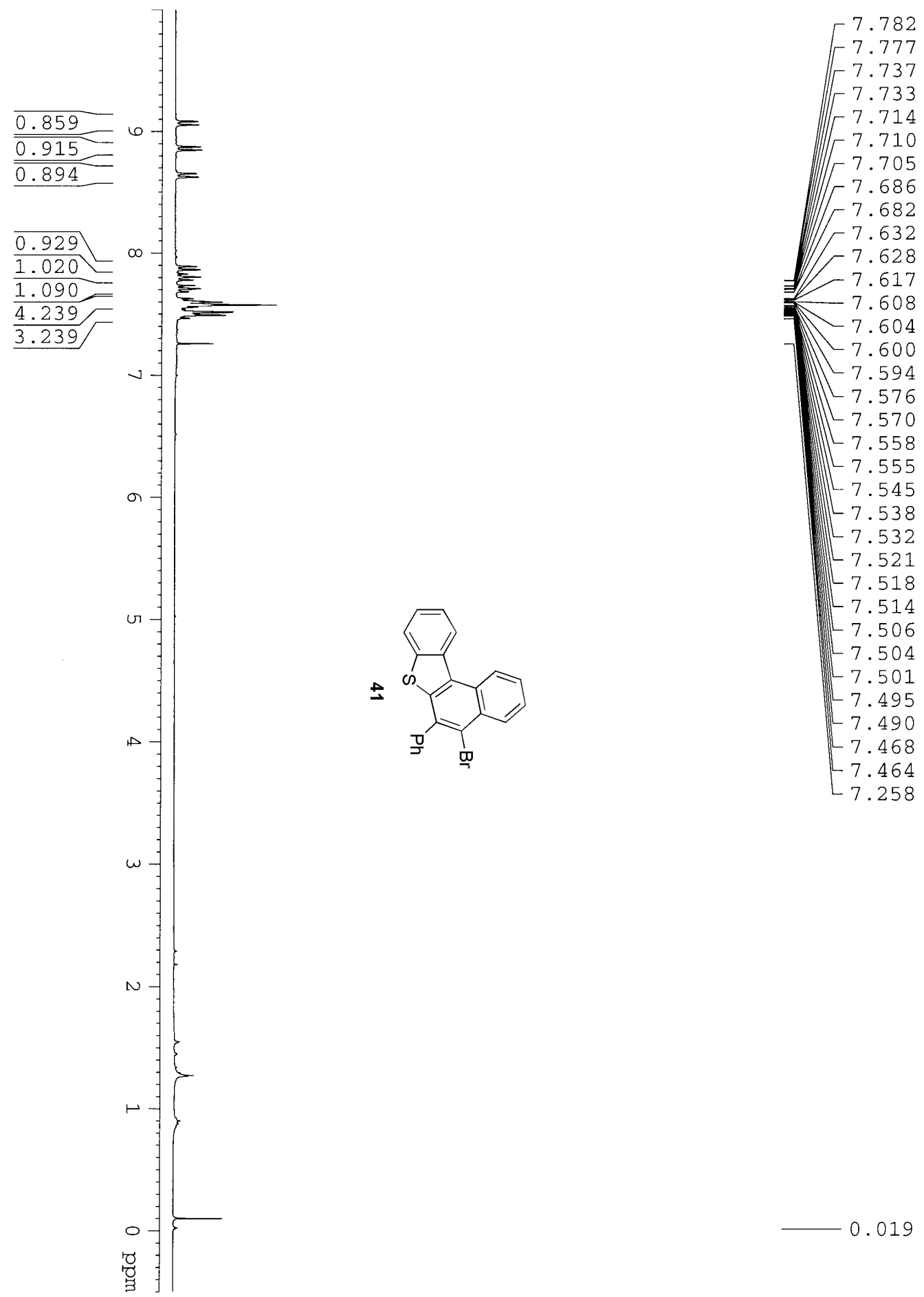

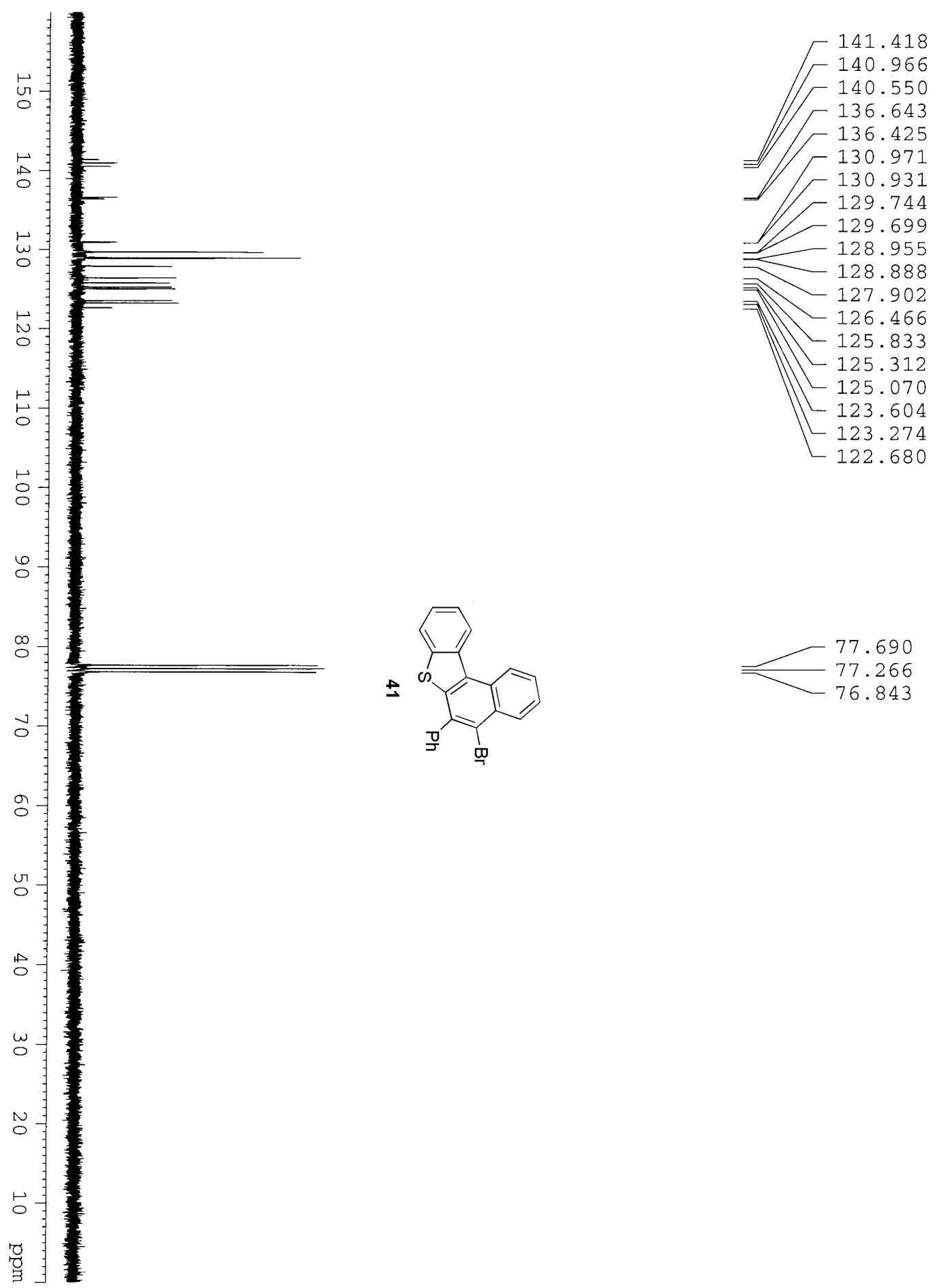

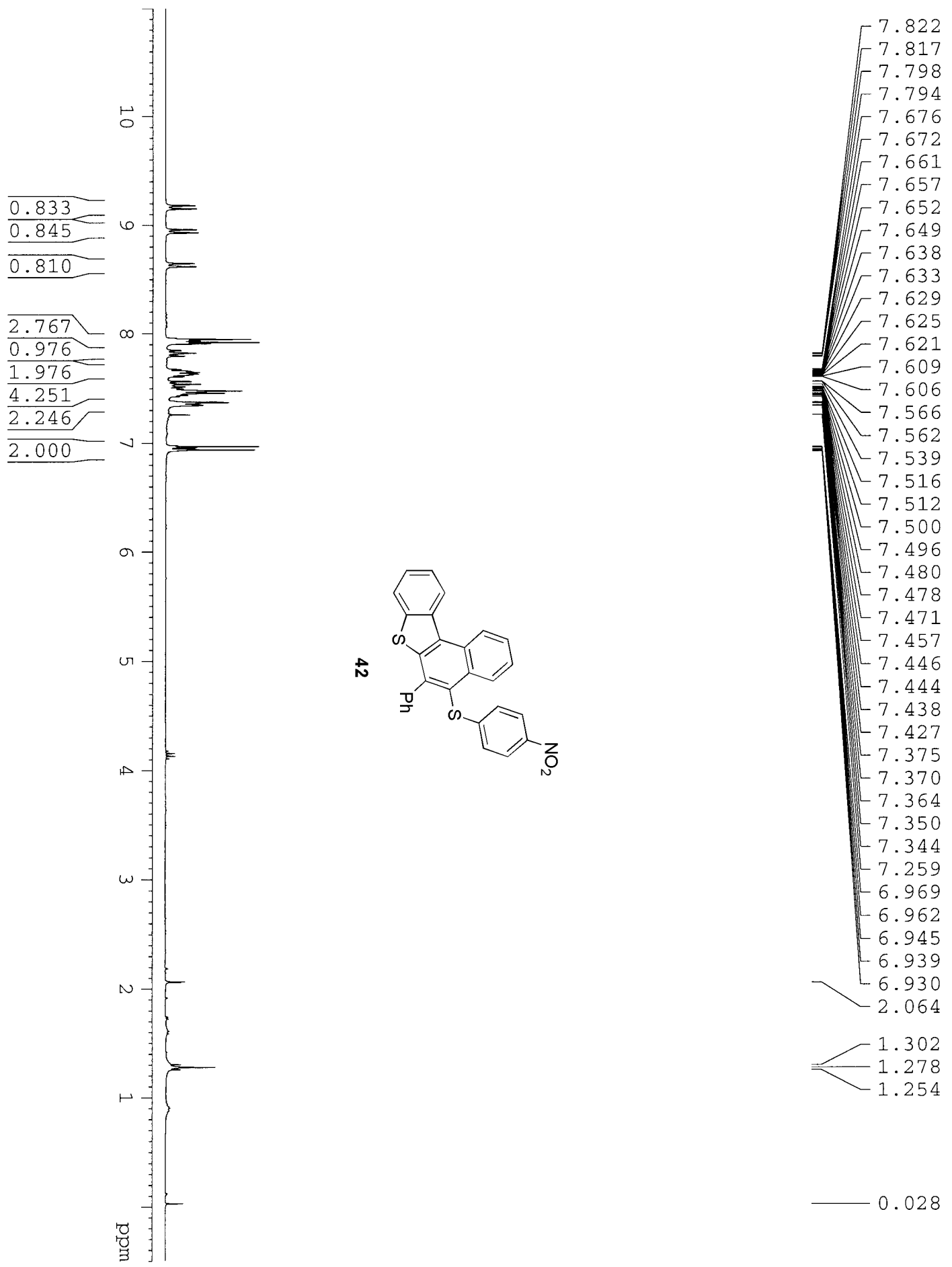

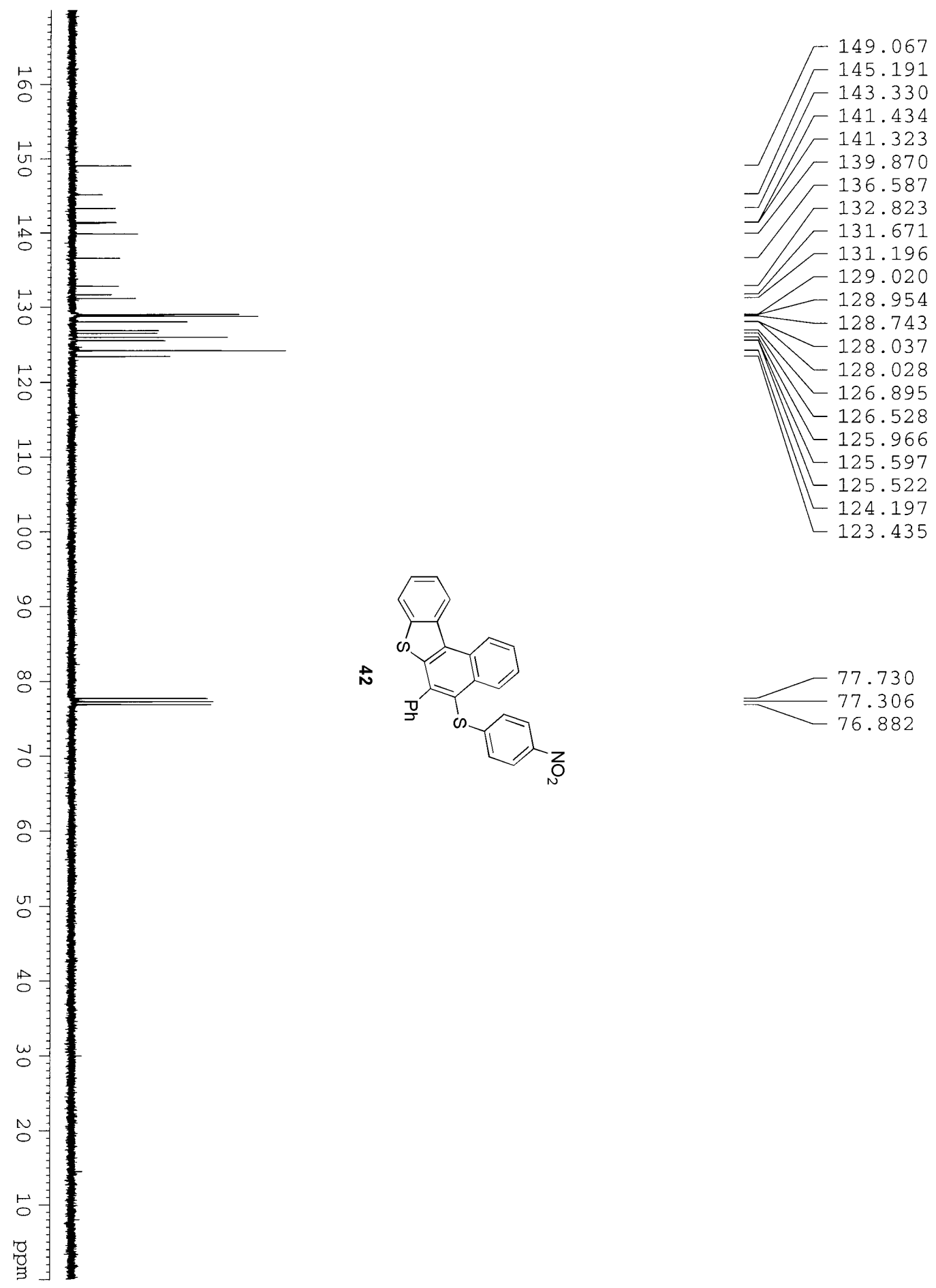

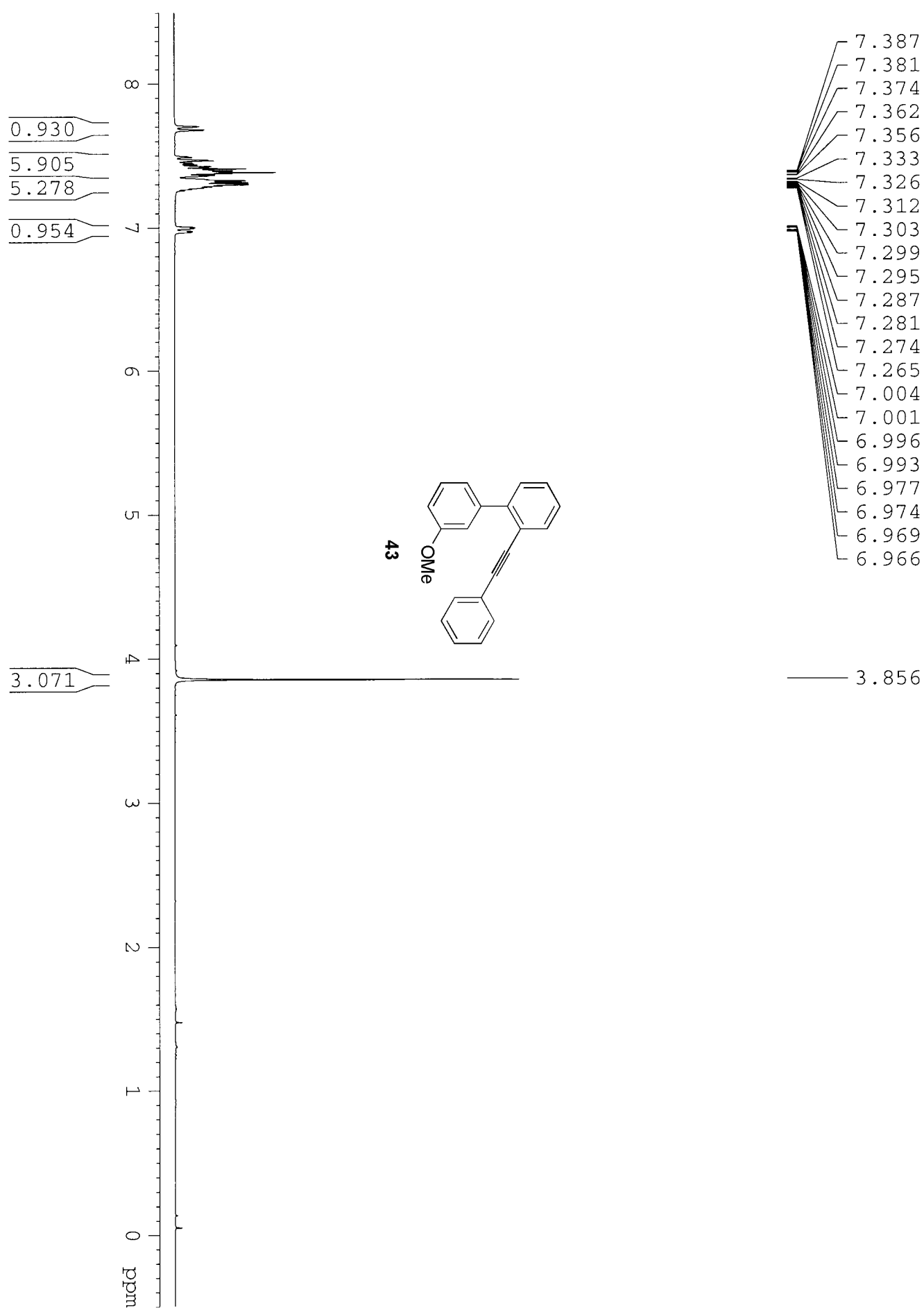

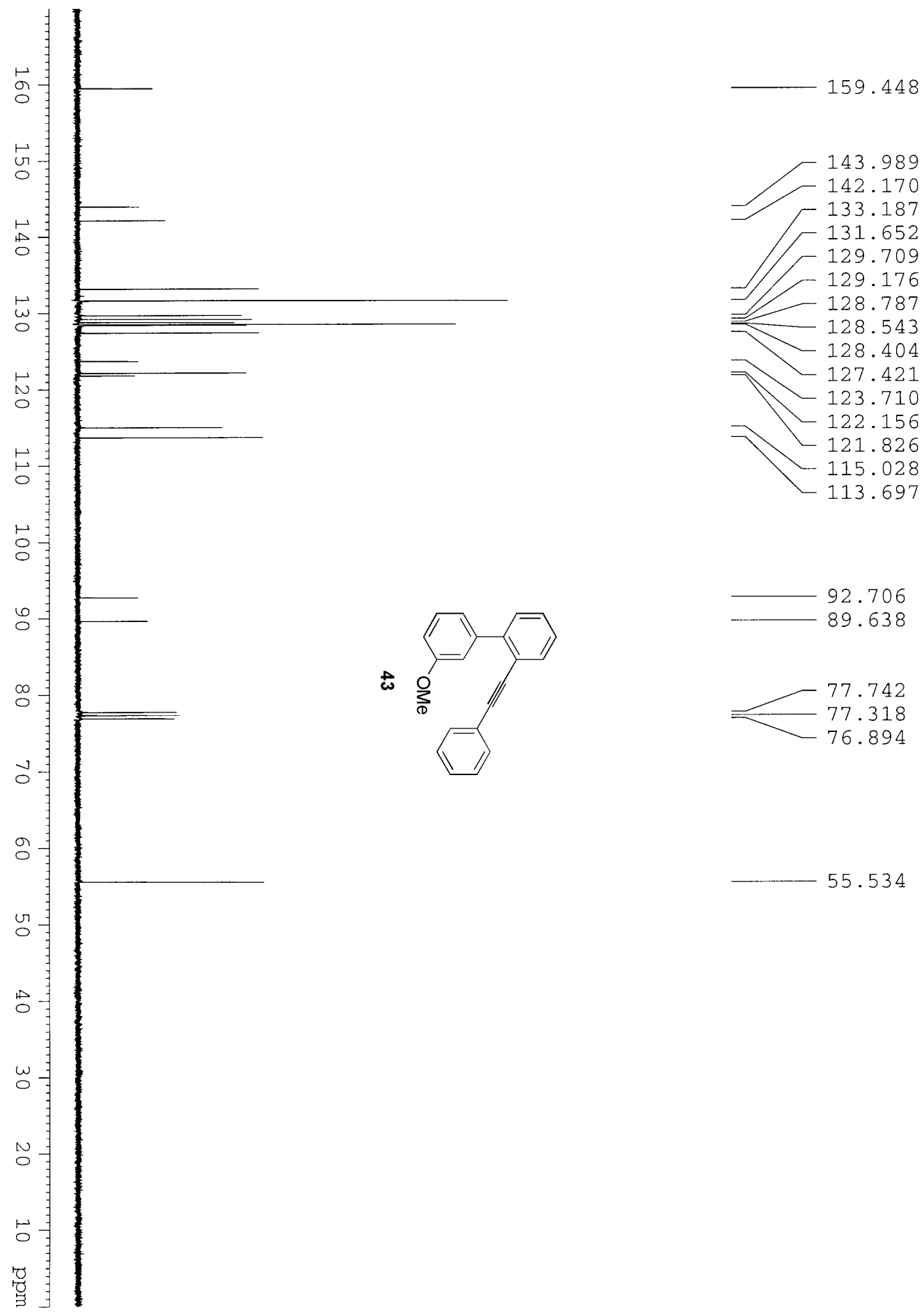

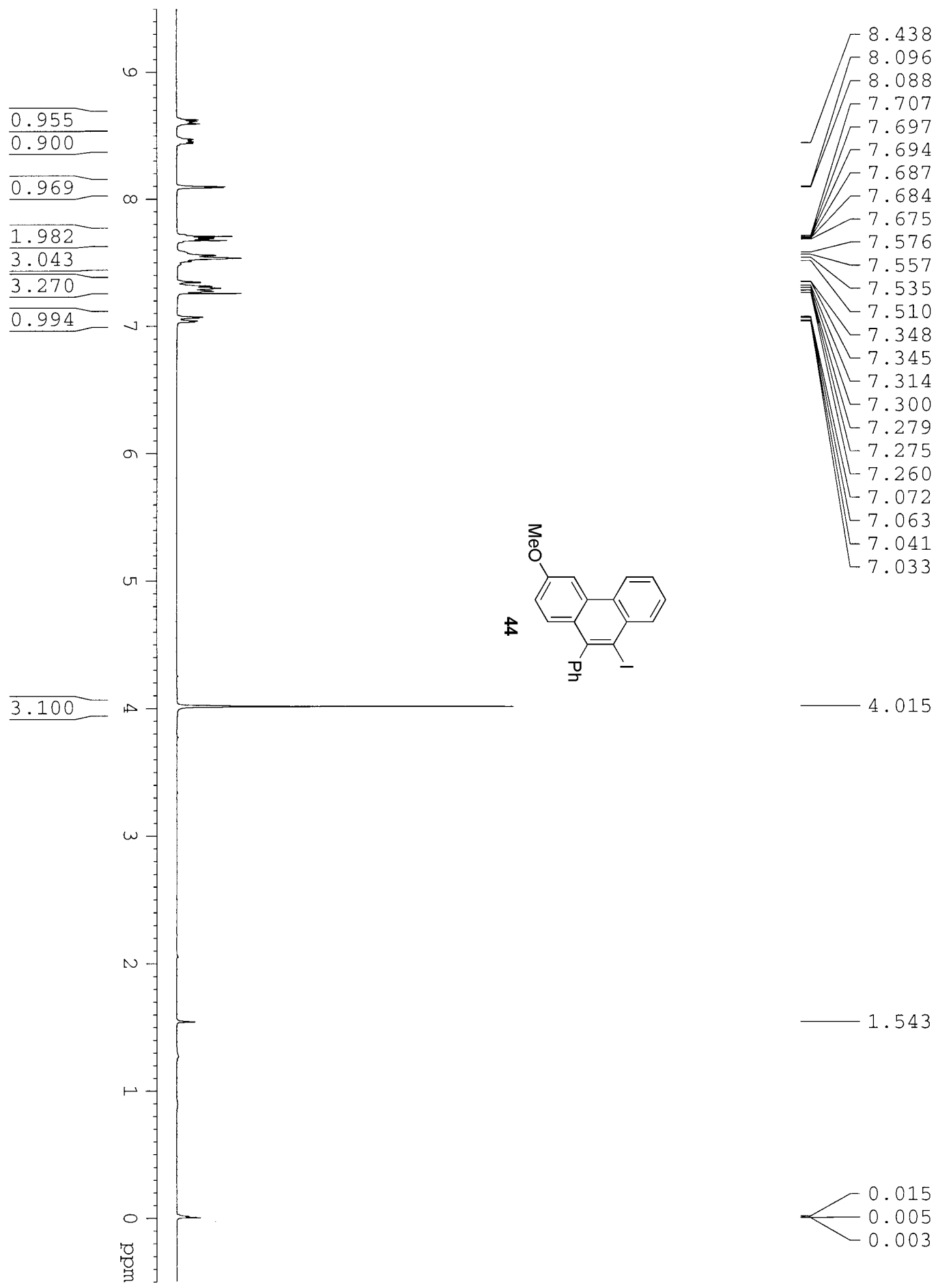


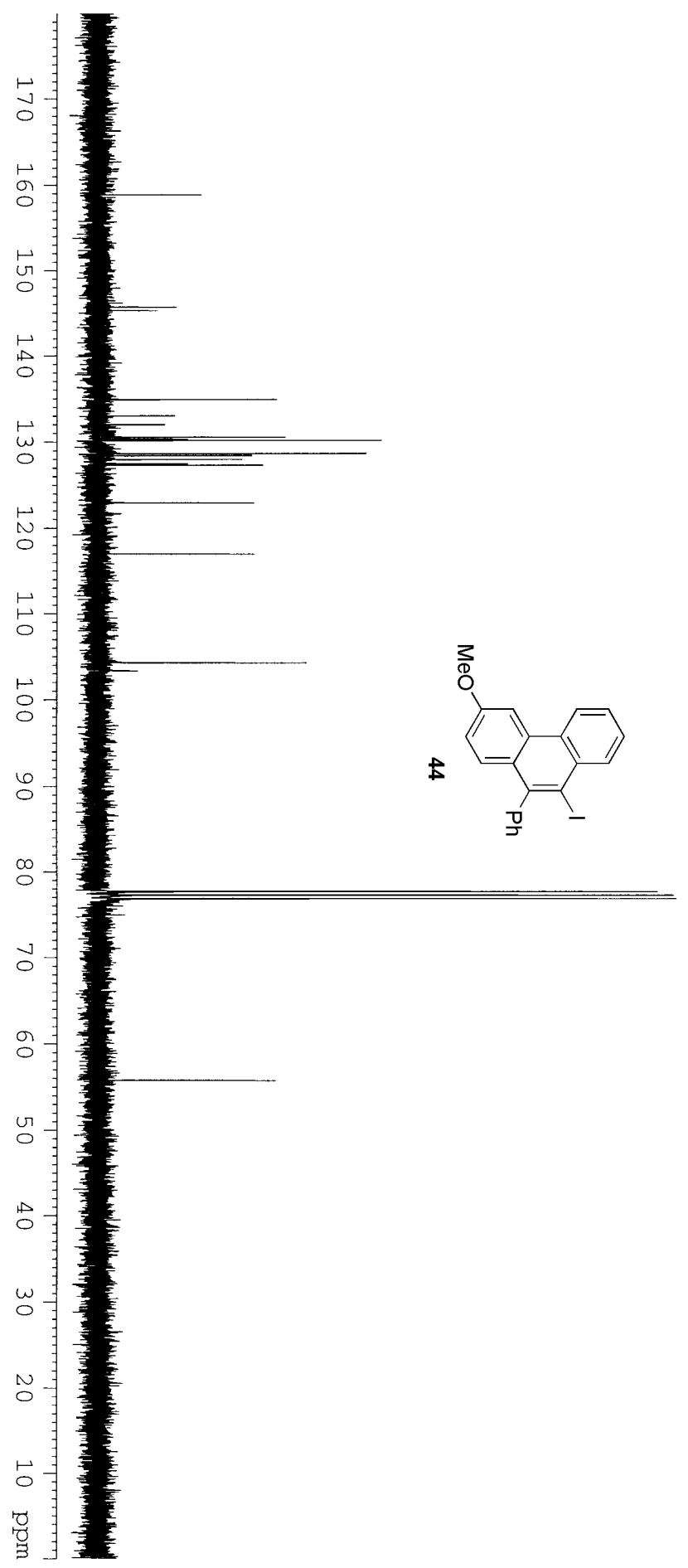

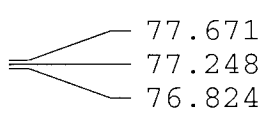

$-55.746$ 

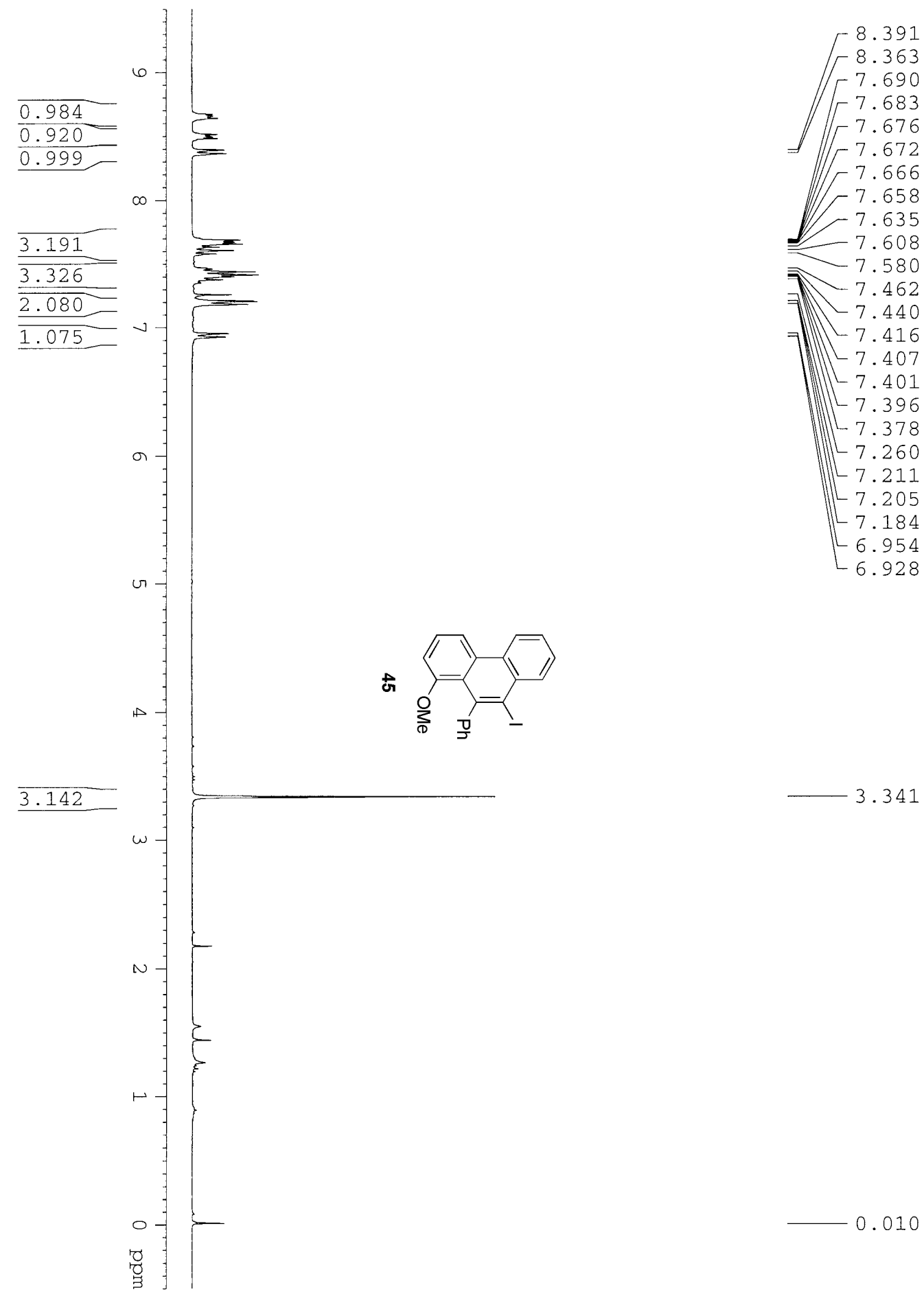

0.010 

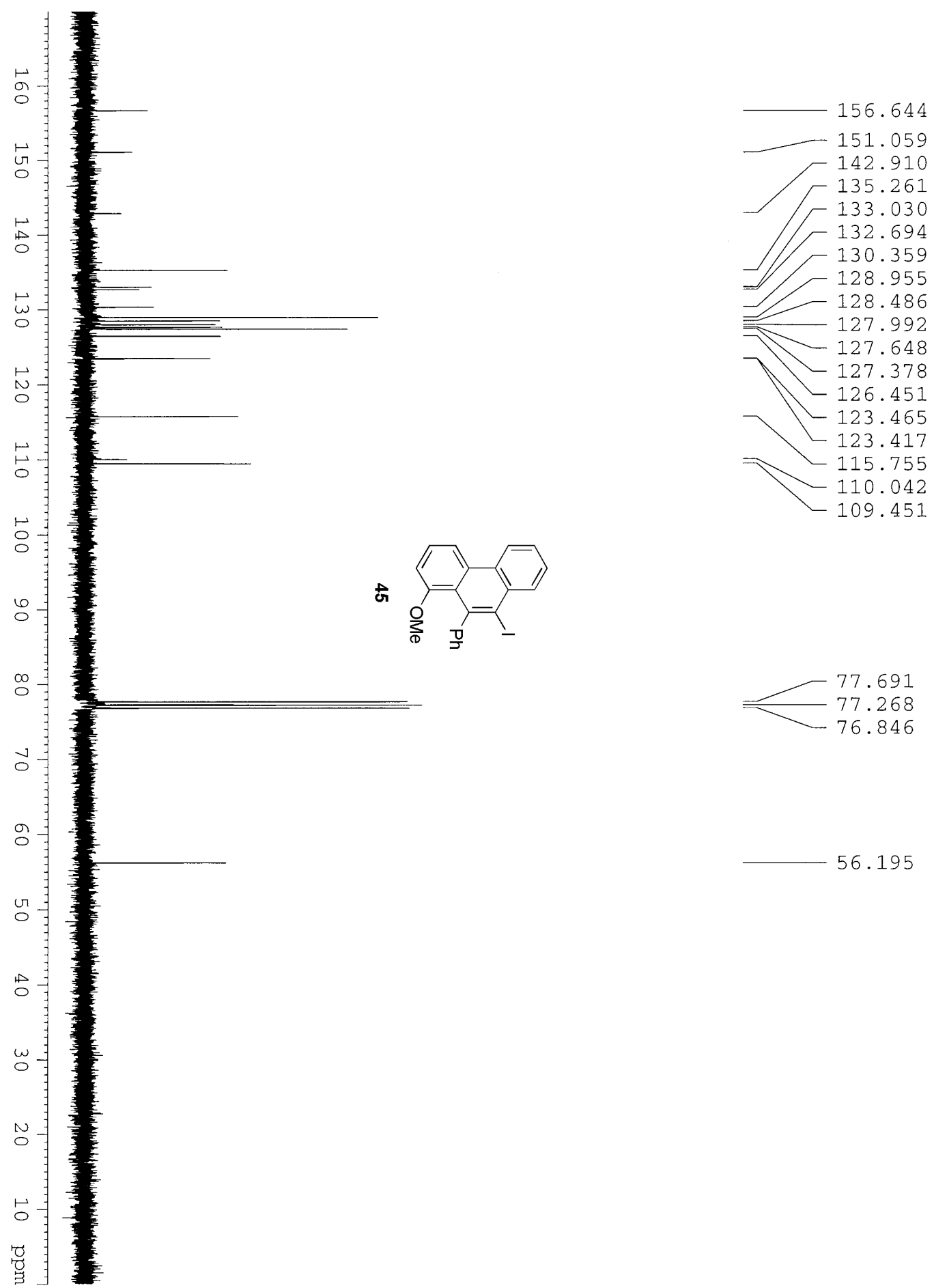

56.195 

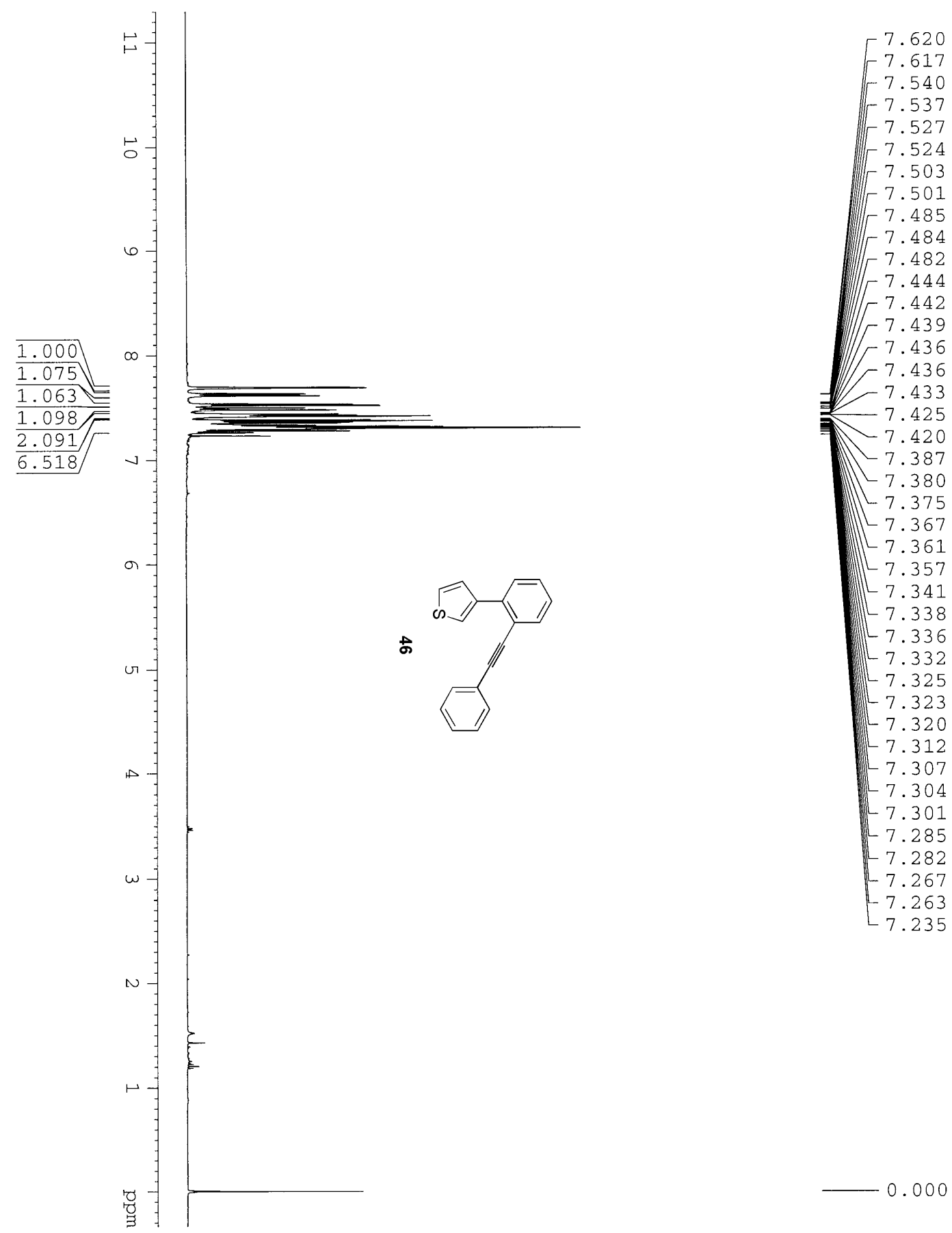


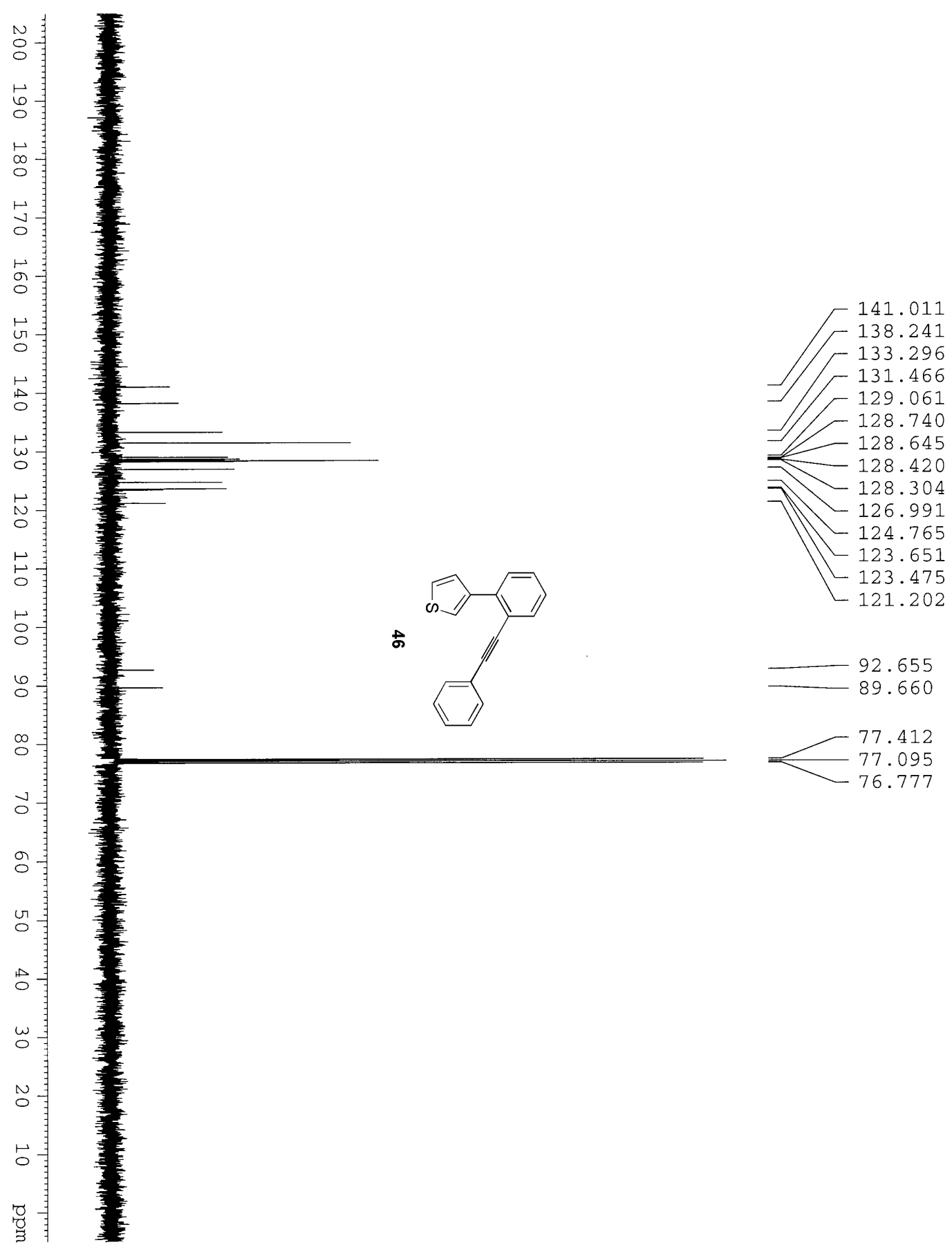



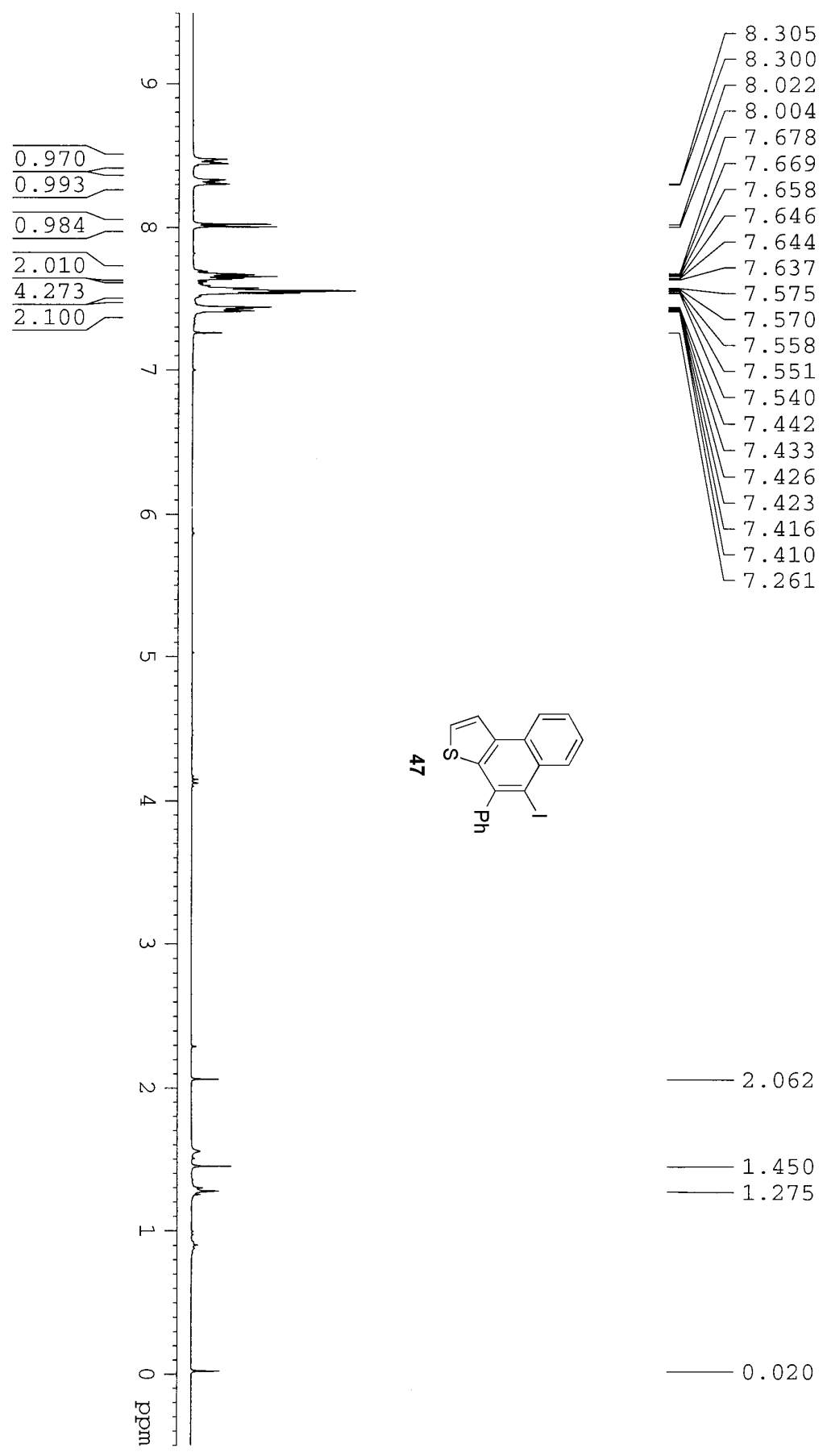

0.020 


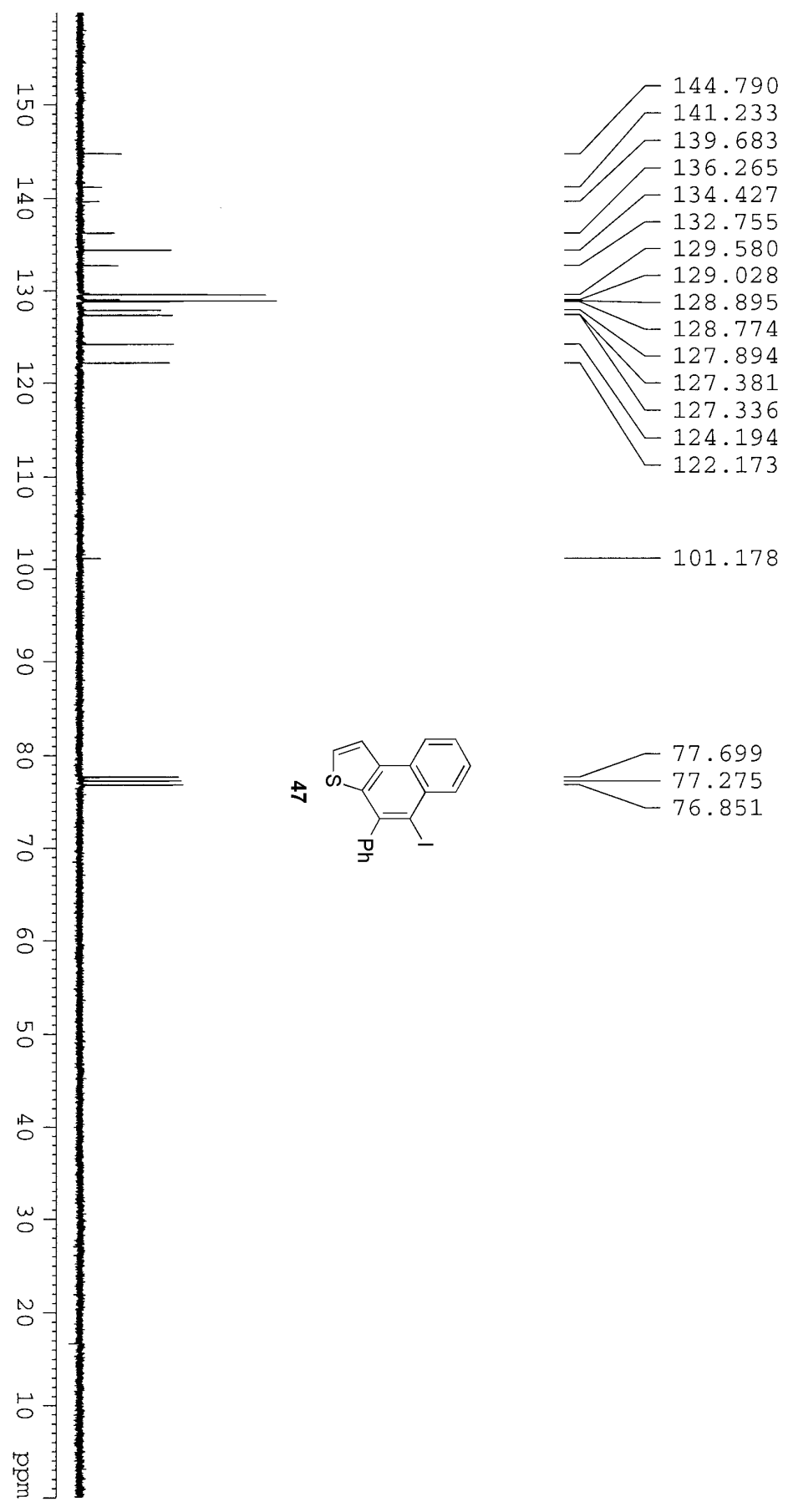



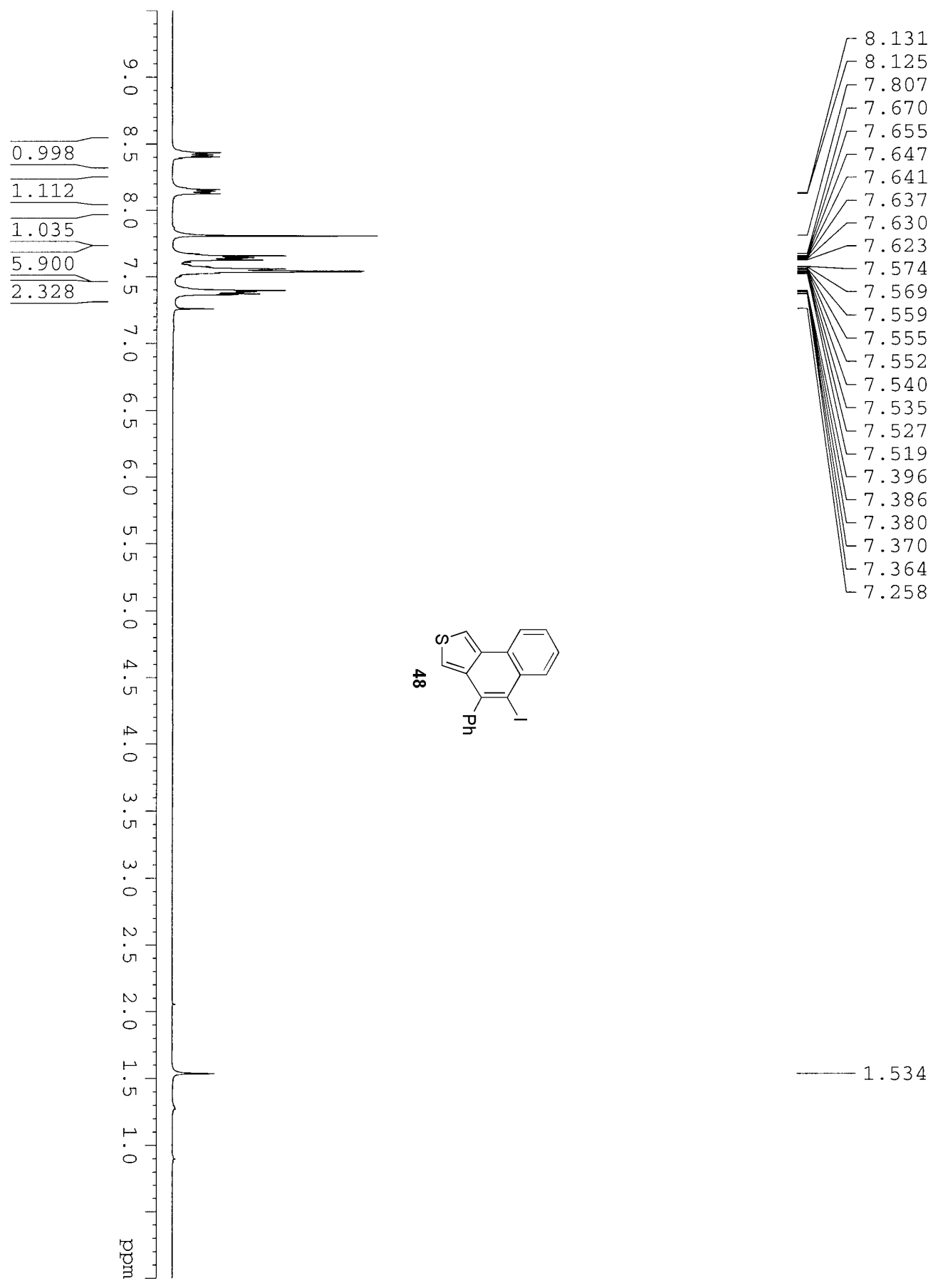

$-\ldots-1.534$ 

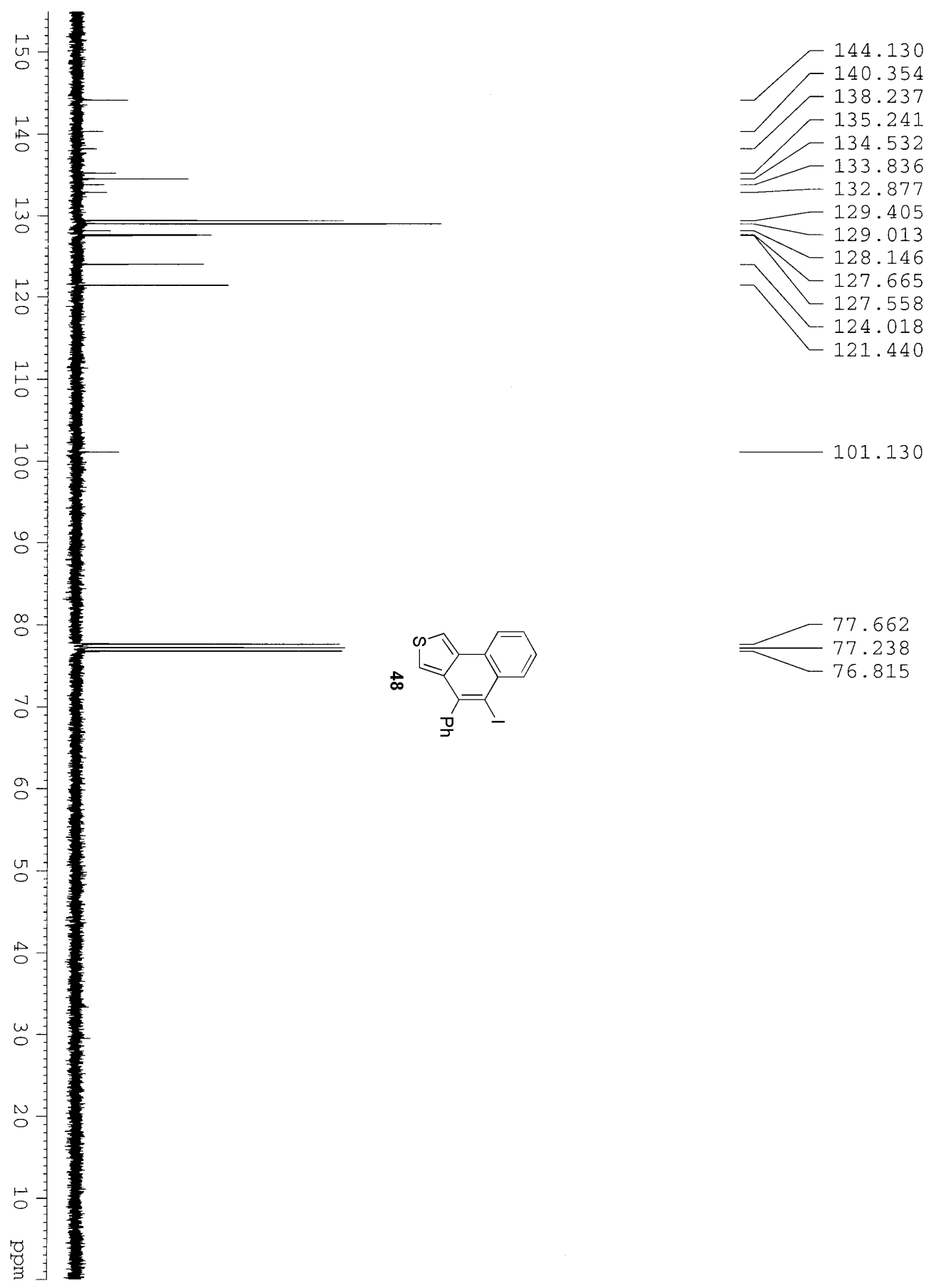

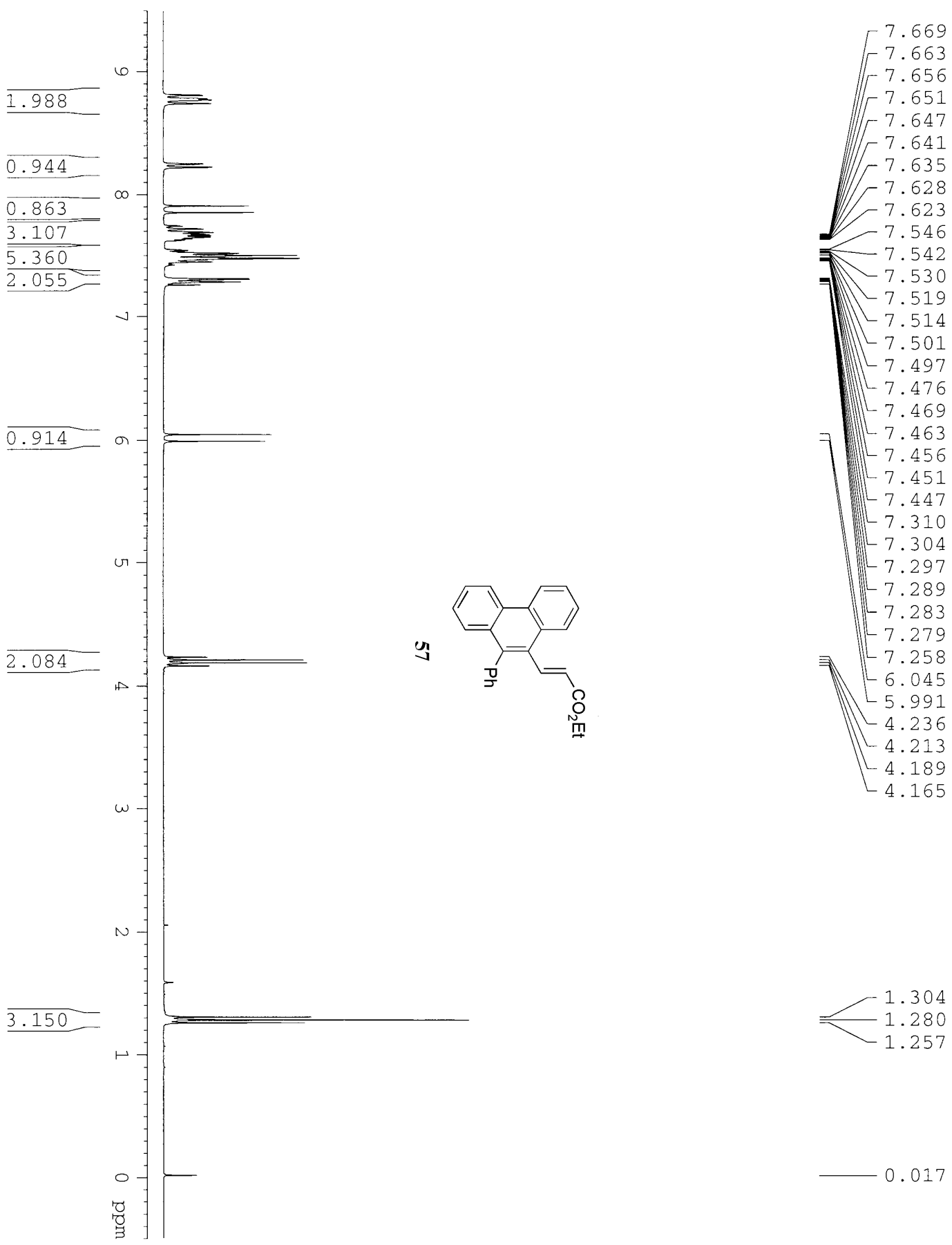

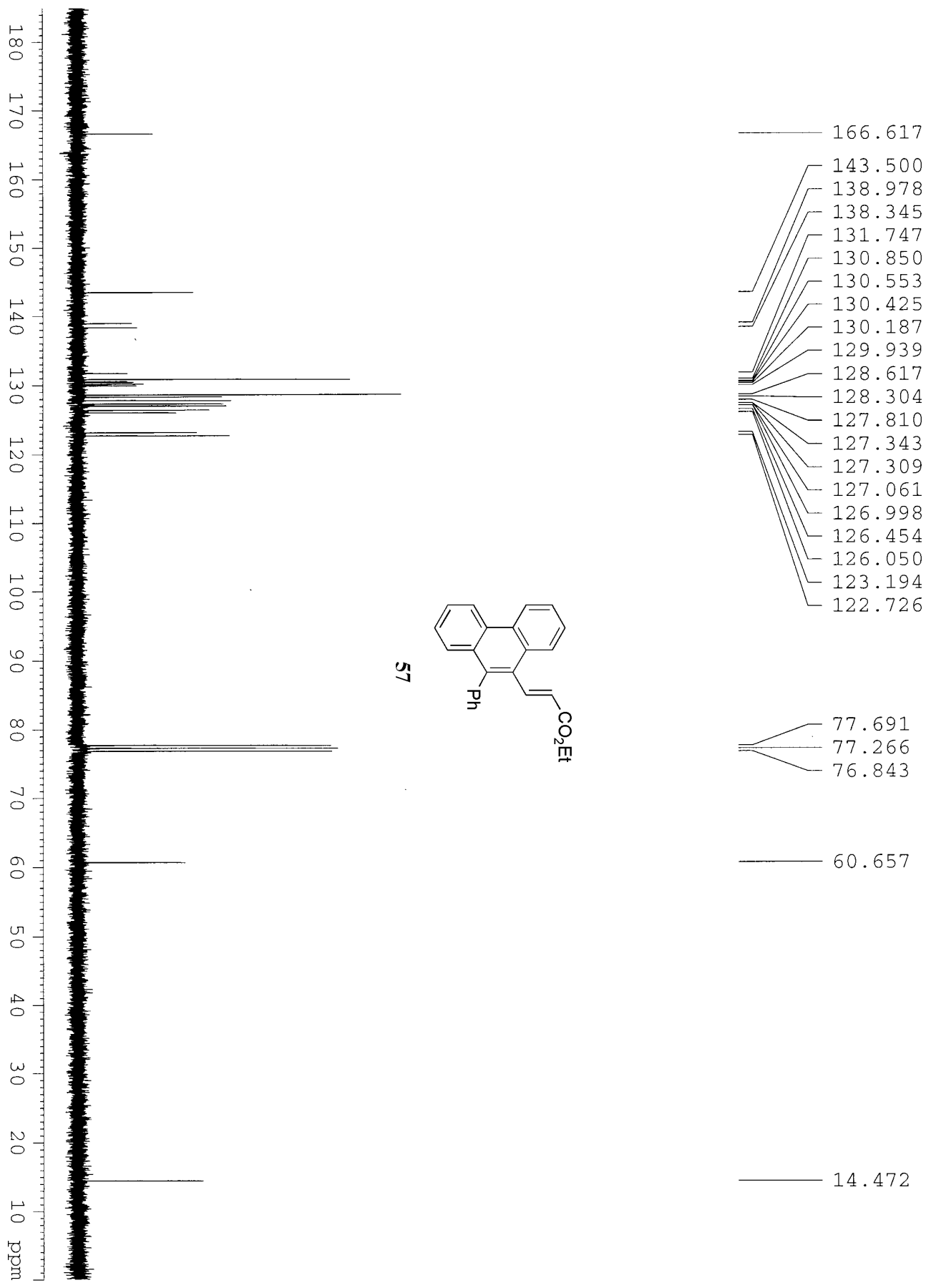

$-60.657$

S60 